\title{
On the Interior Boundary-Value Problem for the Stationary Povzner Equation with Hard and Soft Interactions
}

\author{
VLADISLAV A. PANFEROV
}

\begin{abstract}
The Povzner equation is a version of the nonlinear Boltzmann equation, in which the collision operator is mollified in the space variable. The existence of stationary solutions in $L^{1}$ is established for a class of stationary boundaryvalue problems in bounded domains with smooth boundaries, without convexity assumptions. The results are obtained for a general type of collision kernels with angular cutoff. Boundary conditions of the diffuse reflection type, as well as the given incoming profile, are treated. The method is based on establishing the weak compactness of approximate solutions by using estimates of the entropy production.
\end{abstract}

Mathematics Subject Classification (2000): 82C40 (primary); 35Q72, 45K05 (secondary).

\section{1. - Introduction}

The subject of this study is a modification of the nonlinear Boltzmann equation, introduced by A. Y. Povzner in 1962 [33]. The classical Boltzmann equation, which plays a fundamental role in the physical theory, is also a wellknown source of challenging problems for analysts. Many of the difficulties associated to that equation arise because of the discrepancy between the 'natural' a priori bounds for solutions and the structure of the equation. More specifically, the available bounds for the mass, energy and entropy of solutions are not sufficient to define the collision term in the equation as a function from a suitable function class. As a consequence, in the present theory, solutions corresponding to a general type of initial data have to be defined in a rather weak, renormalized sense (see [2], [14], [18] for a discussion of renormalized and other equivalent forms of solutions).

These difficulties become especially apparent in the analysis of boundaryvalue problems, and particularly so in the stationary case, when, as we discuss

This paper is based on a Ph. D. thesis work [31] done at the Chalmers University of Technology, Göteborg, Sweden.

Pervenuto alla Redazione il 22 marzo 2004 e in forma definitiva il 15 ottobre 2004. 
later, there are fewer a priori estimates available. The source of the problems is mainly in the particular structure of the collision term which involves the product of the solution (which is, a priori, a mass density function with some 'mild' regularity) by its value at a different velocity, but at the same point in the physical space (this corresponds to the assumption that collisions are localized in space). While the local type of the interactions is part of the Boltzmann model (and is also dictated by the Boltzmann-Grad limit) there are other wellknown models of collisions in which the pointwise product is replaced by a different structure. One such model is the Enskog equation for a dense gas of hard spheres [3], [33], [34]. The Povzner equation is another closely related model, in which the pointwise in $x$ product of the mass-density functions is replaced by their tensor product (which thereby allows collisions of particles at different points in the physical space).

The structure of the Povzner collision operator makes it a much more friendly object from the point of view of apriori bounds. This fact was recently used by Arkeryd and Nouri [7] in the context of stationary boundary-value problems. Their results concern $L^{1}$ solutions for steady Povzner equations under rather restrictive assumptions on the collision kernels, in domains with convex geometry. The aim of this paper is to extend the results in [7] to a more general setting. We show that solutions with similar properties can be obtained for 'realistic' collision operators of both 'hard' and 'soft' type, in non-convex domains, for a general class of diffuse reflection operators. We also show a solution of the problem with given incoming profile of the distribution on the boundary.

Apart from the motivation presented by the paper [7], the Povzner equation is an interesting object on its own right. While the model itself was originally not introduced with any specific particle system in mind, a useful interpretation of such an equation was later found by Cercignani [12], who obtained a Povznertype equation in the Grad's limit for a system of 'soft' spheres, interacting at a stochastic distance. Hydrodynamic limits of such a particle system were studied by Lachowicz and Pulvirenti [26], who showed the convergence to the solutions of the Euler system. Finally, an important motivation for Povzner-type equations comes from the particle methods in rarefied gas dynamics. Indeed, in typical numerical schemes collisions occur between particles in cells, and therefore the physical collision process is necessarily delocalized. The limiting equations appearing in the convergence analysis of such schemes also involve spatial delocalization of the collision process [11], [25], [37] (they typically include even stronger mollifications than the Povzner equation).

Stationary problems for nonlinear kinetic equations have been studied extensively in the recent years. Existence and stability results in situations close to equilibrium have been known for a long time [21], [27], [28], [31], [36]. Important results have also been obtained for discrete-velocity models [15], [16] and for the Boltzmann equation for large Knudsen numbers [24], [28]. More recently, stationary solutions for Boltzmann equation in slab geometry, with large data were obtained in measure setting [4], [13] and in $L^{1}$ setting [6], [8]. 
Stationary solutions for the Boltzmann equation in general space dimension, with given incoming profile were recently obtained by Arkeryd [1] and Arkeryd and Nouri [9]. The above results for the Boltzmann equation rely on certain truncations for small speeds, which are not needed in the case of the Povzner equation (the truncation can also be removed in case of one space dimension for the Boltzmann equation). Time-dependent solutions for the Povzner equations have been studied in [10], [23], [33].

The key technique of the paper is based on the weak $L^{1}$ compactness of solutions which is obtained via the entropy production analysis of Arkeryd and Nouri [5], [6], [7], [8]. This approach yields $L^{1}$ solutions which satisfy the equation in the renormalized sense, and it generally does not promise the solutions to be unique. In the 'hard' interactions case an apriori bound in $L^{1}$ for the collision term is available, and the equation is satisfied in a regular weak sense (see Section 2). In the 'soft' interactions case this improved regularity is not available. However, we are able to show that solutions satisfy the iterated integral form as in [2] with 'correct' boundary terms (the boundary conditions are thereby satisfied almost everywhere). To obtain this result we use a recent technique by Mischler [29], which in the present case yields the weak compactness of the boundary traces.

The structure of the paper is as follows. Section 2 presents the model and formulates the main results of the paper (Theorems 2.1-2.3). Section 3 discusses the basic a priori bounds for the total energy and the entropy production of solutions. In Section 4 solutions for a truncated problem are constructed, which will later allow to pass to the limit in the equations and to find solutions of the original problem. Section 5 deals with a priori lower bounds for solutions in domains with nonconvex geometry. Section 6 contains the weak compactness analysis based on the entropy production estimates. The final step establishing the weak compactness in $x$ (Lemma 6.6) is based on an averaging lemma type argument similar to [20] which was obtained by the author independently in [31]. This argument is the key to the result for 'soft' interactions. The last section is devoted to proofs of Theorems 2.1, 2.2 and 2.3.

ACKNOWLEDGEMENTS. I would like to express my gratitude to Leif Arkeryd and Alexei Heintz, for their encouragement and advice. I am also thankful to Anne Nouri, Miroslaw Lachowicz, Irene Gamba and Reinhard Illner who contributed with suggestions and support.

\section{2. - Formulation of the problem and main results}

We study a model problem describing the mass density function $f$ for a gas in a vessel $\Omega$, with generally non-constant boundary temperature. The function $f$ is nonnegative and satisfies the following stationary Povzner equation

$$
v \cdot \nabla_{x} f=Q(f, f), \quad(x, v) \in \Omega \times \mathbb{R}^{3} .
$$


We assume that $\Omega$ is a bounded domain in $\mathbb{R}^{3}$, with smooth boundary $\partial \Omega$. The Povzner collision operator $Q(f, f)$ can be written as follows:

$$
Q(f, f)=Q^{+}(f, f)-Q^{-}(f, f),
$$

where $Q^{-}(f, f)=f v(f)$ is the loss term,

$$
v(f)=\iint_{\Omega \times \mathbb{R}^{3}} f\left(y, v_{*}\right) \chi_{\left\{\left(v-v_{*}\right) \cdot \omega_{x y}<0\right\}} B\left(x, v, y, v_{*}\right) d y d v_{*}
$$

is the integral collision frequency, and $Q^{+}$is the gain term, defined as follows:

$$
Q^{+}(f, f)=\iint_{\Omega \times \mathbb{R}^{3}} f\left(x, v^{\prime}\right) f\left(y, v_{*}^{\prime}\right) \chi_{\left\{\left(v-v_{*}\right) \cdot \omega_{x y}>0\right\}} B\left(x, v, y, v_{*}\right) d y d v_{*}
$$

Here, $\omega_{x y}=(x-y) /|x-y|$, is the unit vector defining the impact parameter, and $v^{\prime}$ and $v_{*}^{\prime}$ denote the pre-collisional velocities, given by the formulas

$$
v^{\prime}=v-\omega_{x y}\left(\omega_{x y} \cdot\left(v-v_{*}\right)\right), \quad v_{*}^{\prime}=v_{*}+\omega_{x y}\left(\omega_{x y} \cdot\left(v-v_{*}\right)\right) .
$$

Notice, that the conditions $\left(v-v_{*}\right) \cdot \omega_{x y}<0$ and $\left(v-v_{*}\right) \cdot \omega_{x y}>0$ in (2.3) and (2.4) correspond to the (physically meaningful) assumptions that particles should be approaching each other before the collision and running away from each other afterwards. We insist on keeping the $\chi$ factors in the definition of the collision terms because of their physical meaning, and also since they will play a crucial role for establishing a priori bounds for the total energy of the solutions.

The kernel $B$ in (2.3) and (2.4) describes the rate of collisions for pairs of particles at points $(x, v)$ and $\left(y, v_{*}\right)$ in the phase space. By its physical meaning it is nonnegative, and satisfies the symmetry relations

$$
B\left(x, v, y, v_{*}\right)=B\left(y, v_{*}, x, v\right)=B\left(x, v^{\prime}, y, v_{*}^{\prime}\right),
$$

expressing the facts that the particles are interchangeable and the collision process is reversible. We allow functions $B$ of the form

$$
B\left(x, v, y, v_{*}\right)=b(x, y) B\left(\omega_{x y}, v-v_{*}\right),
$$

where $b(x, y)$ is a mollifier and $B\left(\omega, v-v_{*}\right)$ is a collision kernel of the Boltzmann equation [14]. More precisely, we assume that $b(x, y)$ is a bounded symmetric function on $\Omega \times \Omega$ which satisfies the following support condition: there are constants $c_{0}>0$ and $c_{1}>0$, such that for all $x \in \Omega$

$$
b(x, y) \geq c_{0}>0, \quad \text { for all } \quad y \in \Omega \cap\left\{|x-y| \leq c_{1}\right\} .
$$


We take the kernel $B\left(\omega, v-v_{*}\right)$ corresponding to the inverse power potentials with Grad's cutoff:

$$
B\left(\omega, v-v_{*}\right)=\left|v-v_{*}\right|^{\beta} h\left(\left|\frac{\left(\omega, v_{*}-v\right)}{\left|v_{*}-v\right|}\right|\right),
$$

where $-3<\beta<2$, and $h$ is a nonnegative function in $L^{\infty}(0,1)$ which for every $\varepsilon>0$ satisfies

$$
h(z) \geq c_{\varepsilon}>0, \quad \text { outside a set of measure } \varepsilon \text { in }(0,1) .
$$

(The condition $h \in L^{\infty}(0,1)$, motivated by the example of 'hard spheres', could be replaced by a less restrictive integral type condition, to the price of introducing additional technical arguments in the proofs.) We distinguish the cases of 'soft' interactions, when $-3<\beta<0$ and 'hard' interactions for $0 \leq \beta<2$ (the case $\beta=0$ is also known as pseudo-maxwellian molecules).

The Povzner collision operator reduces formally to the Enskog one (with the constant density factors) if we set

$$
\beta=1, \quad h(x)=d^{2} x, \quad b(x, y)=\frac{1}{|x-y|} \delta\left(\frac{|x-y|^{2}}{2}-\frac{d^{2}}{2}\right),
$$

where $d=$ const is the diameter of the particles and $\delta$ is the one-dimensional Dirac delta function (the six-fold integration in (2.3) and (2.4) then reduces to a five-fold integration). The Boltzmann operator is obtained by setting $d=0$ in the last of the formulas (2.11).

We assume the boundary $\partial \Omega$ to be a surface of class $C^{1}$ satisfying the following mild regularity condition: for all $\theta \in(0,1)$ there exists $d=d_{\theta}>0$ such that

$$
\begin{gathered}
\text { for all } x \in \partial \Omega \text { the cone } \\
C_{\theta, d}(x)=\left\{x^{\prime}|| x^{\prime}-x\left|\leq d,\left(x^{\prime}-x\right) \cdot n_{x}>\theta\right| x^{\prime}-x \mid\right\} \subseteq \Omega .
\end{gathered}
$$

(The above condition is automatically satisfied, for instance, when the boundary is a Lyapunov surface, see e.g. [22]). The notation $n_{x}$ will be used for the inward normal vector field on $\partial \Omega$. It is convenient to split the boundary in the phase space $\Omega \times \mathbb{R}^{3}$ into two parts: the 'incoming' $\partial \Omega^{+}$and the 'outcoming' $\partial \Omega^{-}$, defined as follows:

$$
\partial \Omega^{ \pm}=\left\{(x, v) \in \partial \Omega \times \mathbb{R}^{3} \mid \pm\left(v \cdot n_{x}\right)>0\right\} .
$$

We will look for nonnegative, integrable solutions of (2.1) for which we would like to specify a priori the total mass or, more generally, the quantity

$$
\iint_{\Omega \times \mathbb{R}^{3}} f(x, v) w(x, v) d x d v,
$$


where $w(x, v)$ is a suitable weight function (for 'hard' forces, it will be convenient to choose $w(x, v)=\left(1+|v|^{2}\right)^{\beta / 2}$, where $\beta$ is the exponent in (2.9)). We shall also assume (and subsequently prove) that solutions have boundary traces (see, e.g. [35]) on $\partial \Omega^{+}$and $\partial \Omega^{-}$, denoted by $f_{+}$and $f_{-}$, respectively, which are integrable with respect to the measure $\left|v \cdot n_{x}\right| d \sigma_{x} d v$, where $d \sigma_{x}$ is the surface area measure on $\partial \Omega$. Integrals with respect to $\left|v \cdot n_{x}\right| d \sigma_{x} d v$ have physical meaning of boundary fluxes.

We study the following two types of boundary conditions. The first one is a condition of diffuse reflection type, formulated as follows:

$$
f_{+}=\mathcal{R} f_{-}, \quad(x, v) \in \partial \Omega^{+},
$$

where $\mathcal{R}$ is a linear integral operator of the following type:

$$
\mathcal{R} f_{-}(x, v)=\left|v \cdot n_{x}\right|^{-1} \int_{w \cdot n_{x}<0} k(x, v, w) f_{-}(x, w)\left|w \cdot n_{x}\right| d w,
$$

for $(x, v) \in \partial \Omega^{+}$. The kernel $k(x, v, w)$ is assumed to be nonnegative and satisfy the following assumptions (cf. [14]).

A) The mass conservation condition

$$
\int_{v \cdot n_{x}>0} k(x, v, w) d v=1
$$

is assumed to hold for all $(x, w) \in \partial \Omega^{-}$.

B) There are two half-space Maxwellians, $M_{\mathrm{w}}^{+}(x, v)$ and $M_{\mathrm{w}}^{-}(x, v)$, with temperatures bounded away from zero and from above, such that

$$
M_{\mathrm{w}}^{+}=\mathcal{R} M_{\mathrm{w}}^{-}, \quad(x, v) \in \partial \Omega^{+} .
$$

Notice that using (2.16) and the homogeneity of (2.14) we can assume, without restricting the generality

$$
\int_{v \cdot n_{x}>0} M_{\mathrm{w}}^{+}(x, v)\left|v \cdot n_{x}\right| d v=\int_{w \cdot n_{x}<0} M_{\mathrm{w}}^{-}(x, w)\left|w \cdot n_{x}\right| d w=1, \quad x \in \Omega .
$$

Therefore, we can write

$$
M_{\mathrm{w}}^{ \pm}(x, v)=\frac{\vartheta_{ \pm}^{2}(x)}{2 \pi} e^{-\frac{1}{2} \vartheta_{ \pm}(x)|v|^{2}},
$$

where $\vartheta_{ \pm}(x)$ are the inverse temperatures of $M_{\mathrm{w}}^{ \pm}$, which may in general be different functions of $x$.

C) Finally, we assume that for every $x \in \partial \Omega, v \cdot n_{x}>0$ and $w \cdot n_{x}<0$,

$$
\left|v \cdot n_{x}\right| \Phi_{1}(|v|) \leq k(x, v, w) \leq\left|v \cdot n_{x}\right| \Phi_{2}(|v|),
$$


where $\Phi_{1}(|v|)$ and $\Phi_{2}(|v|)$ are Maxwellians with positive densities and temperatures. A classical example of the boundary operator $\mathcal{R}$ of the above type is the Maxwellian diffuse reflection [14], in which case $k(x, v, w)=$ $\left|v \cdot n_{x}\right| M_{\mathrm{w}}^{+}(x, v)$.

The second, simpler type of boundary condition allows us to specify the 'incoming profile' of the solution. In this case,

$$
f_{+}(x, v)=\gamma f_{b}(x, v), \quad(x, v) \in \partial \Omega^{+},
$$

where $f_{b}$ is a given function satisfying

$$
\iint_{\partial \Omega^{+}} f_{b}(x, v)\left(1+|v|^{2}+\log f_{b}(x, v)\right)\left|v \cdot n_{x}\right| d \sigma_{x} d v \leq C,
$$

and $\gamma>0$ is a constant, which we have to leave free, since we would like to specify the value for the functional (2.13). This type of boundary conditions was studied in [5], [6], [8].

The transport operator on the left-hand side of (2.1) can be easily inverted by integration along characteristics, which are the straight lines passing through $x$, in the direction of $v$. We set

$$
f^{\sharp}(x, v, s)=f(x+v s, v)
$$

(and often abbreviate the above notation by writing $f^{\sharp}(s)$ or simply $f^{\sharp}$ ) and define the forward and backward stay times $s^{-}$and $s^{+}$according to

$$
s^{ \pm}(x, v)=\inf \left\{s>0 \mid(x \mp s v, v) \in \partial \Omega^{ \pm}\right\} .
$$

Then $v \cdot \nabla_{x} f=\frac{d}{d s} f^{\sharp}$, and we obtain by integration the following mild form

$$
f^{\sharp}(t)=f^{\sharp}(s)+\int_{s}^{t} Q(f, f)^{\sharp}(\tau) d \tau,
$$

for every $(x, v) \in \Omega \times \mathbb{R}^{3}$ and every $-s^{+}<s<t<s^{-}$. Further, using the splitting of $Q(f, f)$ into the gain and loss terms (2.2), we obtain the so-called exponential form,

$$
f^{\sharp}(t)=f^{\sharp}(s) e^{-\int_{s}^{t} v(f)^{\sharp} d \tau}+\int_{s}^{t} Q^{+}(f, f)^{\sharp}(\tau) e^{-\int_{\tau}^{t} v(f)^{\sharp} d u} d \tau,
$$

for $(x, v) \in \Omega \times \mathbb{R}^{3}$ and for $-s^{+}<s<t<s^{-}$. Since the 'gain' operator $Q^{+}(f, f)$ in (2.24) is nonnegative, by setting in (2.24) $t=s^{-}$and $s=-s^{+}$ and denoting $x^{+}=x-s^{+}(x, v) v$ and $x^{-}=x+s^{-}(x, v) v$, we obtain the following inequalities

$$
f_{+}\left(x^{+}, v\right) e^{-\int_{-s^{+}(x, v)}^{0} v(f)^{\sharp} d \tau} \leq f(x, v) \leq f_{-}\left(x^{-}, v\right) e^{\int_{0}^{s^{-}(x, v)} v(f)^{\sharp} d \tau} .
$$


These inequalities are an important tool in the analysis, allowing comparison of the values of the solution inside the domain with the boundary traces.

In order to control the exponential factors in (2.25) it is desirable to have suitable bounds for the collision frequency $v(f)$. In the case of 'hard' forces, when $\beta \geq 0$ in (2.9) and the kernel $B\left(x, v, y, v_{*}\right)$ is locally bounded, a bound in a weighted $L^{\infty}$ space is readily available. More precisely, we have

(2.26) $\sup _{x \in \Omega}\left\{v(f)(x, v)\left(1+|v|^{2}\right)^{-\beta / 2}\right\} \leq C \iint_{\Omega \times \mathbb{R}^{3}} f(x, v)\left(1+|v|^{2}\right)^{\beta / 2} d x d v$.

In the case of 'soft' forces, the collision frequency is generally not locally bounded, but a weaker estimate is available: for every $R>0$,

$$
\int_{|v| \leq R}\left\{\sup _{x \in \Omega} v(f)(x, v)\right\} d v \leq C_{R} \iint_{\Omega \times \mathbb{R}^{3}} f(x, v) d x d v .
$$

The latter estimate can be interpreted as follows (using an argument based on Chebyshev's inequality): for $|v| \leq R$ the collision frequency is bounded uniformly outside a set of velocities of 'small' measure in $\{|v| \leq R\}$.

If the solution $f$ is integrable and satisfies suitable decay conditions for $v$ large, and if in addition $Q(f, f)$ is integrable as a function of $x$ and $v$, we obtain the following integral identity by using Green's formula for the boundary traces (cf. [35]). For every test function $\psi \in L^{\infty}\left(\Omega \times \mathbb{R}^{3}\right)$ such that $v \cdot \nabla_{x} \psi \in$ $L^{\infty}\left(\Omega \times \mathbb{R}^{3}\right)$, we get

$$
\begin{aligned}
& \iint_{\partial \Omega^{-}} f_{-} \psi_{-}\left|v \cdot n_{x}\right| d \sigma_{x} d v-\iint_{\Omega \times \mathbb{R}^{3}} f v \cdot \nabla_{x} \psi d x d v \\
= & \iint_{\partial \Omega^{+}} f_{+} \psi_{+}\left|v \cdot n_{x}\right| d \sigma_{x} d v+\iint_{\Omega \times \mathbb{R}^{3}} Q(f, f) \psi d x d v,
\end{aligned}
$$

where $d \sigma_{x}$ denotes the surface measure on $\partial \Omega$. The above identity can be used to define weak solutions of (2.1) under the condition that $Q(f, f) \in L^{1}\left(\Omega \times \mathbb{R}^{3}\right)$. Namely, we say that a function $f \in L^{1}\left(\Omega \times \mathbb{R}^{3}\right)$ is such a solution if (2.28) holds for every test function $\psi$ satisfying the two above conditions.

However, the integrability of the collision term should not be taken for granted: in fact, we are only able to prove it in the case of 'hard' or pseudoMaxwellian interactions, when $\beta \geq 0$ in (2.9). In the case of 'soft' interactions, when $\beta<0$, we generally only have $v(f) \in L_{\text {loc }}^{1}\left(\Omega \times \mathbb{R}^{3}\right)$, and correspondingly, $Q(f, f) /(1+f) \in L_{\mathrm{loc}}^{1}\left(\Omega \times \mathbb{R}^{3}\right)$. Therefore, the solutions in this case can be defined in the renormalized sense [18], which in fact implies that (2.23) and (2.24) hold 'for almost all characteristics', but which is not enough for the last term in (2.28) to make sense for all $\psi$. Instead of working directly with renormalized solutions in this case we prefer to use a weakened form of (2.28), similar to the one used in [2] which is obtained as follows.

We notice that every characteristic of (2.1) is uniquely determined by specifying a pair $(x, v)$, where $x \in \partial \Omega$ and $v$ satisfies $\left(v \cdot n_{x}\right)<0$ (or $\left(v \cdot n_{x}\right)>0$ : 
this gives a different parameterization). We can therefore identify the characteristics with the points of either $\partial \Omega^{-}$or $\partial \Omega^{+}$. The natural measure on these sets is then $\left|v \cdot n_{x}\right| d \sigma_{x} d v$, and we have the identity

$$
\begin{aligned}
\iint_{\Omega \times \mathbb{R}^{3}} \varphi(x, v) d x d v & =\iint_{\partial \Omega^{-}}\left|v \cdot n_{x}\right| \int_{-s^{+}(x, v)}^{0} \varphi^{\sharp} d s d \sigma_{x} d v \\
& =\iint_{\partial \Omega^{+}}\left|v \cdot n_{x}\right| \int_{0}^{s^{-(x, v)}} \varphi^{\sharp} d s d \sigma_{x} d v,
\end{aligned}
$$

for every $\varphi \in L^{1}\left(\Omega \times \mathbb{R}^{3}\right)$, with $\varphi^{\sharp}$ defined by (2.22).

Arguing as in [2], we can show that if $Q(f, f)^{\sharp}(x, v, \cdot) \in L^{1}\left(\left(-s^{+}, s^{-}\right)\right)$ for almost all $(x, v) \in \Omega \times \mathbb{R}^{3}$, and if the boundary traces are integrable with respect to the measure $\left|v \cdot n_{x}\right| d \sigma_{x} d v$, then (2.23) implies the following iterated integral form

$$
\begin{aligned}
& \iint_{\partial \Omega^{-}} f_{-} \psi_{-}\left|v \cdot n_{x}\right| d \sigma_{x} d v-\iint_{\partial \Omega^{+}} f_{+} \psi_{+}\left|v \cdot n_{x}\right| d \sigma_{x} d v \\
= & \iint_{\Omega \times \mathbb{R}^{3}} f v \cdot \nabla_{x} \psi d x d v+\iint_{\partial \Omega^{-}}\left|v \cdot n_{x}\right| \int_{-s^{+}(x, v)}^{0} Q(f, f)^{\sharp} \psi^{\sharp} d s d \sigma_{x} d v
\end{aligned}
$$

for every $\psi \in L^{\infty}\left(\Omega \times \mathbb{R}^{3}\right)$ for which also $v \cdot \nabla_{x} \psi \in L^{\infty}\left(\Omega \times \mathbb{R}^{3}\right)$. Conversely, if the above identity is true for all $\psi$ satisfying the above conditions, then $f$ satisfies the equation in the mild and the exponential form. Identity (2.30) is thereby a weakened version of (2.28): in (2.30) we do not require integrability of $Q(f, f)$ on $\Omega \times \mathbb{R}^{3}$, but rather the integrability of $\int_{-s^{+}(x, v)}^{0} Q(f, f)^{\sharp} d s$ over the space of characteristics.

We can now formulate the main results of the paper. The first theorem concerns the case of the Povzner equation with 'hard' interactions and diffuse reflection boundary conditions.

THEOREM 2.1. For every $\kappa>0$, equation (2.1) with the kernel of the type (2.7)(2.9) and $0 \leq \beta<2$, has nonnegative weak solutions in the sense of the integral identity (2.28), which satisfy $f\left(1+|v|^{2}\right) \in L^{1}\left(\Omega \times \mathbb{R}^{3}\right), Q(f, f) \in L^{1}\left(\Omega \times \mathbb{R}^{3}\right)$ and

$$
\iint_{\Omega \times \mathbb{R}^{3}} f(x, v)\left(1+|v|^{2}\right)^{\beta / 2} d x d v=\kappa .
$$

Moreover, the boundary traces $f_{ \pm}$are integrable with respect to the measure $(1+$ $\left.|v|^{2}\right)\left|v \cdot n_{x}\right| d \sigma_{x} d v$ and satisfy (2.14) almost everywhere on $\partial \Omega^{+}$.

In the case of 'soft' interactions we obtain solutions in the weaker sense given by the iterated integral form (2.30); however, we do not have to include the moment of order $\beta$ in the normalization condition. The precise formulation is as follows. 
THEOREM 2.2. Under the assumptions of Theorem 2.1 in the case $-3<\beta<0$, for every $\kappa>0$ there exist nonnegative solutions in the sense of identity (2.30), such that $f\left(1+|v|^{2}\right) \in L^{1}\left(\Omega \times \mathbb{R}^{3}\right)$ and

$$
\iint_{\Omega \times \mathbb{R}^{3}} f(x, v) d x d v=\kappa .
$$

In addition, the boundary traces $f_{ \pm}$are integrable with respect to the measure $\left(1+|v|^{2}\right)\left|v \cdot n_{x}\right| d \sigma_{x} d v$ and satisfy (2.14) almost everywhere on $\partial \Omega^{+}$.

Finally, we formulate the results for the incoming profile boundary condition (2.20).

THEOREM 2.3. Let $f_{b}$ be a nonnegative function satisfying conditions (2.21), and assume that there is an $R>0$ such that for every $\varepsilon>0$,

$$
f_{b}\left(x^{+}(x, v), v\right) \geq c_{\varepsilon}>0
$$

pointwise in $x \in \Omega$ and for $v$ outside a set of volume $\varepsilon$ in $\{|v| \leq R\}$. For every $\kappa>0$, there exist nonnegative solutions $f\left(1+|v|^{2}\right) \in L^{1}\left(\Omega \times \mathbb{R}^{3}\right)$ of equation (2.1), which satisfy the normalization condition (2.31) if $0 \leq \beta<2$ and (2.32) if $-3<\beta<0$. The boundary traces $f_{ \pm}$are integrable with respect to the measure $\left(1+|v|^{2}\right) \mid v$. $n_{x} \mid d \sigma_{x} d v$ and satisfy the boundary condition (2.20), with constant $\gamma$ dependent on $\kappa$, almost everywhere on $\partial \Omega^{+}$.

\section{3. - Basic a priori bounds}

The theory of weak solutions for nonlinear kinetic equations is based on using certain 'physical' a priori bounds, namely those expressing conservation of mass, momentum, energy, and the entropy production [14]. In general, only some of the bounds applying to the time-dependent case give meaningful information about solutions of the steady problem. One especially notable 'omission' is the lack of simple a priori bounds for the total mass in terms of the boundary data, which is not surprising given the structure of the equation, but which makes the analysis significantly harder. In what follows fundamental role will be played by the entropy production functional, which is to be expected, since it determines the time-evolution of a Lyapunov functional in the timedependent case. Another important bound may be obtained in a limit of the time-evolution of the functional $\iint_{\Omega \times \mathbb{R}^{3}}(x \cdot v) f d x d v$ (we provide a more direct argument that applies in the steady case in Lemma 3.1). This bound provides an a priori estimate for the total kinetic energy.

The following weak form of the collision operator will play the key role in establishing the a priori bounds (and indeed it may be accepted as an alternative 
definition of the collision term). For every suitable test function $\psi(x, v)$ we have

$$
\begin{aligned}
& \iint_{\Omega \times \mathbb{R}^{3}} Q(f, f) \psi d x d v \\
= & \iint_{\left(\Omega \times \mathbb{R}^{3}\right)^{2}} f f_{*}\left(\psi^{\prime}-\psi\right) \chi_{\left\{\left(v-v_{*}\right) \cdot \omega_{x y}<0\right\}} B d y d v_{*} d x d v,
\end{aligned}
$$

where we used shorthand notations $f=f(x, v), \psi=\psi(x, v), f_{*}=f\left(y, v_{*}\right)$, and $\psi^{\prime}=\psi\left(x, v^{\prime}\right)$. The above weak form is obtained by the change of variables $\left(v, v_{*}\right) \mapsto\left(v^{\prime}, v_{*}^{\prime}\right)$ in the integral corresponding to $Q^{+}(f, f)$. Performing another change of variables, $\left(x, v, y, v_{*}\right) \mapsto\left(y, v_{*}, x, v\right)$ and using the invariance of the product $f f_{*}$ and the $\chi$ function, we obtain the following symmetrized version of (3.1):

$$
\frac{1}{2} \iint_{\left(\Omega \times \mathbb{R}^{3}\right)^{2}} f f_{*}\left(\psi^{\prime}+\psi_{*}^{\prime}-\psi-\psi_{*}\right) \chi_{\left\{\left(v-v_{*}\right) \cdot \omega_{x y}<0\right\}} B d y d v_{*} d x d v,
$$

where we also set $\psi_{*}^{\prime}=\psi\left(x, v_{*}^{\prime}\right)$ and $\psi_{*}=\psi\left(x, v_{*}\right)$. Setting $\psi=1, v_{i}$ and $|v|^{2}$, where $v_{i}, i=1,2,3$, denote the components of the velocity vector, we see that, formally, the collision term $Q(f, f)$ satisfies the global in $x$ conservation of mass, momentum and energy (in fact, the conservation of mass holds locally, as can be observed from (3.1)).

Another important idea that we will use below is to introduce an (artificial) absorption term in the equation, considering the following modified version of (2.1):

$$
v \cdot \nabla_{x} f+\alpha f=Q(f, f),
$$

where $\alpha>0$ is a constant. Solutions of the original problem will be obtained by taking the limit $\alpha \rightarrow 0$ in (3.3). Equation (3.3) is in certain aspects 'nicer' than the original equation (2.1), and can be used to obtain solutions of (2.1) by letting $\alpha$ tend to zero. This approach has also been used in [1], [6], [9].

The presence of the absorption with $\alpha>0$ will modify the mass balance; therefore, we account for this in the boundary condition (in the diffuse reflection case) by replacing (2.14) by the following modified version:

$$
f_{+}=\mathcal{R} f_{-}+g, \quad(x, v) \in \partial \Omega^{+} .
$$

Here $g=g(x, v)$ is a source term proportional to the total mass, which is defined as follows

$$
g(x, v)=\alpha S(x, v) \iint_{\Omega \times \mathbb{R}^{3}} f d x d v, \quad(x, v) \in \partial \Omega^{+},
$$

and $S$ is a bounded smooth function on $\partial \Omega^{+}$, rapidly decaying for $|v|$ large, which satisfies

$$
\iint_{\partial \Omega^{+}} S(x, v)\left|v \cdot n_{x}\right| d \sigma_{x} d v=1 .
$$

Our first result is the following bound for the total energy of the solutions. 
Lemma 3.1. Assume that $f$ is a sufficiently regular solution of (3.3) with $\alpha \geq 0$, such that $f\left(1+|v|^{2}\right) \in L^{1}\left(\Omega \times \mathbb{R}^{3}\right)$ and $Q(f, f)\left(1+|v|^{2}\right) \in L^{1}\left(\Omega \times \mathbb{R}^{3}\right)$. Then

$$
\begin{aligned}
& \iint_{\Omega \times \mathbb{R}^{3}} f|v|^{2} d x d v+\iint_{\Omega \times \mathbb{R}^{3}} Q(f, f)(x \cdot v) d x d v \\
\leq & D \iint_{\partial \Omega^{+}} f_{+}\left(1+|v|^{2}\right)\left|v \cdot n_{x}\right| d \sigma_{x} d v,
\end{aligned}
$$

where $D$ is the diameter of $\Omega$.

REMARK.

1) We notice at once that the second term on the left-hand side of the above estimate is nonnegative (it is identically zero in the case of the Boltzmann equation). To see this, use the symmetrized form (3.2) and notice that

$$
\begin{aligned}
x \cdot v^{\prime}+y \cdot v_{*}^{\prime}-x \cdot v-y \cdot v_{*} & =-(x-y) \omega_{x y}\left(\omega_{x y} \cdot\left(v-v_{*}\right)\right) \\
& =-|x-y|\left(\omega_{x y} \cdot\left(v-v_{*}\right)\right) \geq 0,
\end{aligned}
$$

for the 'physical' collisions that satisfy $\left(v-v_{*}\right) \cdot \omega_{x y}<0$ (cf. also [19, page 295]).

2) The term with the integral of the collision operator was overlooked in [7]: their argument in Section 4 is incomplete and could be fixed either in the manner of this paper (notice that then the collision term and entropy production functional have to be modified) or by estimating the remainder term (which would require changing the normalization of solutions to account for an extra power of $|v|$ ).

For the reader's convenience we give a complete proof of the estimate in the lemma.

ProOF. Using the weak form (2.28) with $\psi=(x \cdot v)$ we obtain

$$
\begin{aligned}
\iint_{\Omega \times \mathbb{R}^{3}} f|v|^{2} d x d v & +\iint_{\Omega \times \mathbb{R}^{3}} Q(f, f)(x \cdot v) d x d v \\
= & \alpha \iint_{\Omega \times \mathbb{R}^{3}} f(x \cdot v) d x d v \\
& +\iint_{\partial \Omega^{-}} f_{-}(x \cdot v)\left|v \cdot n_{x}\right| d \sigma_{x} d v \\
& -\iint_{\partial \Omega^{+}} f_{+}(x \cdot v)\left|v \cdot n_{x}\right| d \sigma_{x} d v .
\end{aligned}
$$

Using the global momentum conservation, for every $x_{0} \in \Omega$ fixed, we can replace $x$ in every term on the right-hand side by $x-x_{0}$. The right-hand side of (3.7) can then be estimated as

$$
D \iint_{\partial \Omega^{+}} f_{+}\left(1+|v|^{2}\right)\left|v \cdot n_{x}\right| d \sigma_{x} d v
$$


by using the inequality

$$
|(e \cdot v)| \leq \frac{1}{2}\left(1+|v|^{2}\right), \quad \text { with } \quad e=\frac{x-x_{0}}{\left|x-x_{0}\right|},
$$

and the relation

$$
\begin{aligned}
\iint_{\partial \Omega^{-}} f_{-}\left(1+|v|^{2}\right)\left|v \cdot n_{x}\right| d \sigma_{x} d v & +\alpha \iint_{\Omega \times \mathbb{R}^{3}} f\left(1+|v|^{2}\right) d x d v \\
= & \iint_{\partial \Omega^{+}} f_{+}\left(1+|v|^{2}\right)\left|v \cdot n_{x}\right| d \sigma_{x} d v,
\end{aligned}
$$

which follows by applying the mass and energy conservation in the weak form (2.28) with $\psi=1+|v|^{2}$.

REMARK. The right-hand side of the estimate of the lemma can be simplified further in the case of the diffuse reflection boundary condition (3.4) (or the original form (2.14)). Indeed, as a consequence of the mass conservation we have

$$
\iint_{\partial \Omega^{+}} f_{+}\left|v \cdot n_{x}\right| d \sigma_{x} d v=\iint_{\partial \Omega^{-}} f_{-}\left|v \cdot n_{x}\right| d \sigma_{x} d v+\alpha \iint_{\Omega \times \mathbb{R}^{3}} f d x d v,
$$

and using (2.19) and estimating the $|v|^{2}$-moment of $S$ by a constant we obtain

$$
\begin{aligned}
\iint_{\partial \Omega^{+}} f_{+}|v|^{2}\left|v \cdot n_{x}\right| d \sigma_{x} d v & \leq C_{1} \iint_{\partial \Omega^{-}} f_{-}\left|v \cdot n_{x}\right| d \sigma_{x} d v+C_{2} \alpha \iint_{\Omega \times \mathbb{R}^{3}} f d x d v \\
& \leq C \iint_{\partial \Omega^{+}} f_{+}\left|v \cdot n_{x}\right| d \sigma_{x} d v .
\end{aligned}
$$

Thus, the term with the energy inflow on the right hand side of the estimate of the lemma can be estimated by the mass inflow.

We further look for the bounds of the entropy $\iint_{\Omega \times \mathbb{R}^{3}} f \log f d x d v$ and the entropy production functional $\iint_{\Omega \times \mathbb{R}^{3}} e(f, f) d x d v$, where

$$
e(f, f)=\frac{1}{2} \iint_{\Omega \times \mathbb{R}^{3}} f f_{*}\left(\frac{f^{\prime} f_{*}^{\prime}}{f f_{*}}-\log \frac{f^{\prime} f_{*}^{\prime}}{f f_{*}}-1\right) \chi_{\left\{\left(v-v_{*}\right) \cdot \omega_{x y}<0\right\}} B d y d v_{*}
$$

is the local entropy production (in the Enskog equation case (2.11), for the time-dependent problem, this functional determines the evolution of an entropy functional of the type introduced by Résibois [33], see also [3]). We notice that the integrand in (3.8) is nonnegative, and moreover, using (3.2) and the elementary inequality $t \log (s / t) \leq s-t$, we find

$$
\begin{aligned}
& \iint_{\Omega \times \mathbb{R}^{3}} Q(f, f) \log f d x d v \\
= & \frac{1}{2} \iint_{\left(\Omega \times \mathbb{R}^{3}\right)^{2}} f f_{*} \log \frac{f^{\prime} f_{*}^{\prime}}{f f_{*}} \chi_{\left\{\left(v-v_{*}\right) \cdot \omega_{x y}<0\right\}} B d x d v d y d v_{*} \\
\leq & \frac{1}{2} \iint_{\left(\Omega \times \mathbb{R}^{3}\right)^{2}}\left(f^{\prime} f_{*}^{\prime}-f f_{*}\right) \chi_{\left\{\left(v-v_{*}\right) \cdot \omega_{x y}<0\right\}} B d x d v d y d v_{*} .
\end{aligned}
$$


Using this property in the weak form (2.28) with $\psi=\log f$ we obtain

$$
\begin{aligned}
& \iint_{\Omega \times \mathbb{R}^{3}} e(f, f) d x d v+\alpha \iint_{\Omega \times \mathbb{R}^{3}} f \log f d x d v \\
& \quad+\iint_{\partial \Omega^{-}} f_{-} \log f_{-}\left|v \cdot n_{x}\right| d \sigma_{x} d v \leq \iint_{\partial \Omega^{+}} f_{+} \log f_{+}\left|v \cdot n_{x}\right| d \sigma_{x} d v
\end{aligned}
$$

which implies uniform in $\alpha$ bounds for the entropy production and the entropy outflow and, for $\alpha>0$, an $\alpha$-dependent bound for the entropy, provided we have control of the entropy inflow.

Inequality (3.9) is sufficient to provide the desired bounds for the solutions (and the boundary traces) in the case of the given inflow boundary condition (2.20). In the diffuse reflection case (2.14) the term with the entropy inflow in (3.9) generally has to be estimated; a simple and elegant approach that allows one to do so is based on the Darrozes-Guiraud inequality [17]. To this end, set $\Psi(s)=s \log s$ and define the Darrozes-Guiraud information (cf. [29]):

$$
\mathcal{E}_{M_{\mathrm{w}}}(f)=\mathcal{R}\left(M_{\mathrm{w}}^{-} \Psi\left(\frac{f_{-}}{M_{\mathrm{w}}^{-}}\right)\right)-M_{\mathrm{w}}^{+} \Psi\left(\frac{f_{+}-g}{M_{\mathrm{w}}^{+}}\right), \quad(x, v) \in \partial \Omega^{+}
$$

Then $\mathcal{E}_{M_{\mathrm{w}}}(f)$ is a nonnegative quantity, as can be easily verified by applying Jensen's inequality to the function $\Psi(s)$ with the measure

$$
k(x, v, w) \frac{\left|w \cdot n_{x}\right| M_{\mathrm{w}}^{-}(x, w)}{\left|v \cdot n_{x}\right| M_{\mathrm{w}}^{+}(x, v)} d w
$$

which satisfies the unit measure condition in view of (2.17). We then establish the following a priori estimate.

Lemma 3.2. Assume that $f$ is a sufficiently regular solution of (3.3) with $\alpha \geq 0$, such that $f\left(1+|v|^{2}+\log f\right) \in L^{1}\left(\Omega \times \mathbb{R}^{3}\right)$ and $Q(f, f)\left(1+|v|^{2}+\log f\right) \in L^{1}(\Omega \times$ $\left.\mathbb{R}^{3}\right)$. Assume also that $f$ satisfies the boundary condition (3.4) with the boundary operator (2.15) satisfying the conditions (2.16)-(2.19) and with $g$ bounded and having two moments in $L^{1}\left(\partial \Omega^{+},\left|v \cdot n_{x}\right| d \sigma_{x} d v\right)$. Then

$$
\begin{aligned}
& \iint_{\Omega \times \mathbb{R}^{3}} e(f, f) d x d v+\alpha \iint_{\Omega \times \mathbb{R}^{3}} f \log f d x d v \\
& \quad+\iint_{\partial \Omega^{+}} \mathcal{E}_{M_{\mathrm{w}}}(f)\left|v \cdot n_{x}\right| d \sigma_{x} d v \leq C\left(1+\iint_{\partial \Omega^{+}} f_{+}\left|v \cdot n_{x}\right| d \sigma_{x} d v\right),
\end{aligned}
$$

with the constant $C$ depending on $M_{\mathrm{w}}^{+}, M_{\mathrm{w}}^{-}$and the bounds of $g$ only. 
Remark. The estimate of the lemma remains in effect also for $\alpha=0$, in which case we do not have an estimate for the entropy, but we retain the bounds for the entropy production and the Darrozes-Guiraud information.

Proof. Multiplying (3.3) by $\log f$ and integrating over $\Omega \times \mathbb{R}^{3}$ yields

$$
\begin{aligned}
& \iint_{\Omega \times \mathbb{R}^{3}} e(f, f) d x d v+\alpha \iint_{\Omega \times \mathbb{R}^{3}} f \log f d x d v \\
= & \iint_{\partial \Omega^{+}} f_{+} \log f_{+}\left|v \cdot n_{x}\right| d \sigma_{x} d v-\iint_{\partial \Omega^{-}} f_{-} \log f_{-}\left|v \cdot n_{x}\right| d \sigma_{x} d v \\
= & \iint_{\partial \Omega^{+}} M_{\mathrm{w}}^{+} \Psi\left(\frac{f_{+}-g}{M_{\mathrm{w}}^{+}}\right)\left|v \cdot n_{x}\right| d \sigma_{x} d v-\iint_{\partial \Omega^{-}} M_{\mathrm{w}}^{-} \Psi\left(\frac{f_{-}}{M_{\mathrm{w}}^{-}}\right)\left|v \cdot n_{x}\right| d \sigma_{x} d v \\
& +\iint_{\partial \Omega^{+}} M_{\mathrm{w}}^{+} \Psi\left(\frac{f_{+}}{M_{\mathrm{w}}^{+}}\right)\left|v \cdot n_{x}\right| d \sigma_{x} d v-\iint_{\partial \Omega^{+}} M_{\mathrm{w}}^{+} \Psi\left(\frac{f_{+}-g}{M_{\mathrm{w}}^{+}}\right)\left|v \cdot n_{x}\right| d \sigma_{x} d v \\
& +\iint_{\partial \Omega^{+}} f_{+} \log M_{\mathrm{w}}^{+}\left|v \cdot n_{x}\right| d \sigma_{x} d v-\iint_{\partial \Omega^{-}} f_{-} \log M_{\mathrm{w}}^{-}\left|v \cdot n_{x}\right| d \sigma_{x} d v,
\end{aligned}
$$

where $\Psi(s)=s \log s$. We next see that the difference of the first two terms on the right-hand side equals

$$
-\iint_{\partial \Omega^{+}} \mathcal{E}_{M_{\mathrm{W}}}(f)\left|v \cdot n_{x}\right| d \sigma_{x} d v .
$$

To estimate the logarithms of the boundary Maxwellians we recall (2.18) and write

$$
\left|\log M_{\mathrm{w}}^{ \pm}\right| \leq \frac{1}{2} \vartheta_{ \pm}(x)|v|^{2}+2\left|\log \vartheta_{ \pm}(x)\right| \leq C_{1}\left(1+|v|^{2}\right),
$$

where $C_{1}$ is a constant depending on the maximum and minimum of $\vartheta_{ \pm}(x)$. Then, the last two terms in the above identity are estimated as

$$
\begin{aligned}
& C_{1}\left(\iint_{\partial \Omega^{+}} f_{+}\left(1+|v|^{2}\right)\left|v \cdot n_{x}\right| d \sigma_{x} d v+\iint_{\partial \Omega^{-}} f_{-}\left(1+|v|^{2}\right)\left|v \cdot n_{x}\right| d \sigma_{x} d v\right) \\
\leq & 2 C_{1} \iint_{\partial \Omega^{+}} f_{+}\left(1+|v|^{2}\right)\left|v \cdot n_{x}\right| d \sigma_{x} d v \\
\leq & C\left(\iint_{\partial \Omega^{+}} f_{+}\left|v \cdot n_{x}\right| d \sigma_{x} d v+\iint_{\partial \Omega^{+}} g\left(1+|v|^{2}\right)\left|v \cdot n_{x}\right| d \sigma_{x} d v\right),
\end{aligned}
$$

the last inequality following because of the condition (2.16). For the remaining two terms we apply the elementary inequalities

$$
y \log y-(y-s) \log (y-s) \leq s(1+\log y),
$$


for all $y>s$ and $1+\log x \leq x$, for $x>0$. Setting $y=f_{+} / M_{\mathrm{w}}^{+}$and $s=g / M_{\mathrm{w}}^{+}$ we get

$$
\begin{aligned}
& \iint_{\partial \Omega^{+}} M_{\mathrm{w}}^{+} \Psi\left(\frac{f_{+}}{M_{\mathrm{w}}^{+}}\right)\left|v \cdot n_{x}\right| d \sigma_{x} d v-\iint_{\partial \Omega^{+}} M_{\mathrm{w}}^{+} \Psi\left(\frac{f_{+}-g}{M_{\mathrm{w}}^{+}}\right)\left|v \cdot n_{x}\right| d \sigma_{x} d v \\
\leq & \iint_{\partial \Omega^{+}} g\left(1+\log \frac{f_{+}}{M_{\mathrm{w}}^{+}}\right)\left|v \cdot n_{x}\right| d \sigma_{x} d v \leq \iint_{\partial \Omega^{+}} g\left(f_{+}-\log M_{\mathrm{w}}^{+}\right)\left|v \cdot n_{x}\right| d \sigma_{x} d v \\
\leq & C_{3} \iint_{\partial \Omega^{+}} f_{+}\left|v \cdot n_{x}\right| d \sigma_{x} d v+C_{1} \iint_{\partial \Omega^{+}} g\left(1+|v|^{2}\right)\left|v \cdot n_{x}\right| d \sigma_{x} d v \\
\leq & C\left(1+\iint_{\partial \Omega^{+}} f_{+}\left|v \cdot n_{x}\right| d \sigma_{x} d v\right),
\end{aligned}
$$

where $C_{3}$ and $C$ are constants depending on sup $g$ and $\iint_{\partial \Omega^{+}} g\left(1+|v|^{2}\right) \mid v$. $n_{x} \mid d \sigma_{x} d v$. Gathering the above estimated we complete the proof of the lemma.

The structure of the a priori bounds obtained in the previous two lemmas prompts the following general remarks. The integral quantities associated to the solutions in the volume (such as the total energy and the total entropy production) are controlled by the boundary inflows (the total inflow of the mass, in case of the diffuse reflection boundary condition). Therefore, a 'natural' formulation of the problem suggested by these bounds would be to look for solutions $f(x, v)$ which satisfy the condition of prescribed data on the 'incoming' part of the boundary. However, there seems to be an intrinsic difficulty associated with this approach, since by prescribing the boundary data one generally cannot control the total mass of the solutions. In particular, in such a formulation we cannot exclude the possibility of concentrations of mass for small velocities (the rest of the velocity space can be accounted for using the energy estimate). Such a difficulty does not appear if we prescribe the total mass of the solutions, or its weighted analog (2.31), but in that case we need to find a way to control the boundary inflows appearing in the a priori bounds. For this purpose, in [7] a certain transformation involving the $x$ variable and the total mass of the solutions was used. Here we shall apply the following simple (and more general) argument based on homogeneity.

Suppose that for given $\alpha \geq 0$ and $S$ satisfying (3.6), equation (3.3) with the boundary condition (3.4) admits a solution $f(x, v)$ which, for certain $\kappa>0$, satisfies

$$
\mu(f)=\iint_{\Omega \times \mathbb{R}^{3}} f\left(1+|v|^{2}\right)^{\beta^{\prime} / 2} d x d v=\kappa,
$$

where $\beta^{\prime}=\max (0, \beta)$, and such that for certain $\lambda>0$,

$$
\iint_{\partial \Omega^{+}} f_{+}\left|v \cdot n_{x}\right| d \sigma_{x} d v=\lambda
$$


By taking $F(x, v)=f(x, v) / \lambda$ we then get a function with the unit total mass inflow which also satisfies $\mu(F)=\kappa / \lambda$. We also notice that, by homogeneity, $F$ solves the following boundary-value problem

$$
\begin{aligned}
v \cdot \nabla_{x} F+\alpha F & =\frac{\kappa}{\mu(F)} Q(F, F), \quad(x, v) \in \Omega \times \mathbb{R}^{3} \\
F_{+} & =\mathcal{R} F_{-}+\alpha S \iint_{\Omega \times \mathbb{R}^{3}} F d x d v, \quad(x, v) \in \partial \Omega^{+} .
\end{aligned}
$$

Conversely, suppose that for every $\kappa>0$ we are able to find a solution of the problem (3.12), with $\mu(F)>0$, subject to the condition

$$
\iint_{\partial \Omega^{+}} F_{+}\left|v \cdot n_{x}\right| d \sigma_{x} d v=1 .
$$

Then the function $f=\kappa F / \mu(F)$ solves the problem (3.3), (3.4) and satisfies the normalizing condition (3.11). The total inflow for the solution $f$ is then, for fixed $\kappa$, inversely proportional to the weighted mass of solution to the auxiliary problem (3.12), since we have $\lambda=\kappa / \mu(F)$.

The new formulation (3.12) allows us to avoid some of the difficulties mentioned above. In particular, the normalization condition (3.13) implies that the bounds in Lemma 3.1 and 3.2 are expressed in terms of absolute constants, and the normalizing factor $\kappa / \mu(F)$ in front of the collision term controls the collision frequency in view of estimates (2.26) and (2.27). We shall therefore work in this setting, constructing in next section approximating solutions to the problem (3.12) by introducing certain truncations. Instead of the problem of controlling the boundary traces we shall then encounter a problem of controlling the total mass of the normalized solutions $F$. A solution of this problem will be achieved in Sections 5 and 6. We shall focus on the case of diffuse reflection boundary conditions (2.14): the same methodology applies to the problem with the incoming profile condition (2.20), and we postpone the necessary modifications until the proof of Theorem 2.3 in Section 7. In the sequel we shall take $\kappa=1$ in (3.12), without restricting generality.

\section{4. - Solutions of the truncated equations}

Following standard methodology, we introduce certain truncations in the equations, which will allow us to use monotone convergence and fixed point arguments to construct solutions of the problem. Passing to the limit with the truncation parameters and using compactness arguments will then allow us to obtain solutions of the original problem. As a first step, we take in (3.12), $\alpha>0$, fix $r>0$ and $m>0$ and replace the collision rate function $B\left(x, v, y, v_{*}\right)$ in (2.3) and (2.4) by the truncated version

$$
B_{r m}\left(x, v, y, v_{*}\right)=\min \left(m, B\left(x, v, y, v_{*}\right)\right) \chi_{r}\left(x, v, y, v_{*}\right),
$$


where $\chi_{r}$ is a cut-off function defined as 0 if $\min \left\{|v|,\left|v_{*}\right|,\left|v^{\prime}\right|,\left|v_{*}^{\prime}\right|\right\} \leq r$ and 1 otherwise. Thus, the aim of this section will be to find solutions to the problem (3.12), with $\kappa=1$, subject to the normalizing condition (3.13), and with the collision operator $Q_{r m}(F, F)$ obtained by taking (4.1) as a collision rate function. For definiteness, let us take $S$ in (3.12) to be the boundary Maxwellian $M_{\mathrm{w}}^{+}$, so all conditions of Lemma 3.2 are satisfied. In all the sequel we shall systematically denote families and sequences of solutions either by a single letter $(F, f$, etc.) or by a single superscript index, specifying the dependence on the rest of the parameters only when it is necessary.

We further introduce additional truncation parameters $j>0, i>0$ and $l>0$ and for given $\lambda>0, \mu>0$ satisfying $\lambda+\alpha \mu=1$, and given nonnegative functions $f \in L^{1}\left(\Omega \times \mathbb{R}^{3}\right)$ and $g \in L^{1}\left(\partial \Omega^{-},\left|v \cdot n_{x}\right| d \sigma_{x} d v\right)$ consider the following problem of linear transport type (a nonlinearity will be introduced by truncation):

$$
\begin{aligned}
v \cdot \nabla_{x} F+\left(\alpha+v_{j}(f)\right) F & =\varphi_{l} * Q_{j}^{+}(F, f), & & (x, v) \in \Omega \times \mathbb{R}^{3}, \\
F_{+} & =\lambda \mathcal{R}_{i} g+\alpha \mu S, & & (x, v) \in \partial \Omega^{+} .
\end{aligned}
$$

Here $\varphi_{l}=\varphi_{l}(v)$ is a centered Maxwellian with density one and temperature $1 / l$; $v_{j}(f)$ is the regularized collision frequency obtained by replacing $B$ in (2.3) by a regularized collision rate function $B_{r m j}$, such that

$$
\begin{array}{ccc}
B_{r m j}=0 & \text { if } & \max \left(|v|,\left|v_{*}\right|,\left|v^{\prime}\right|,\left|v_{*}^{\prime}\right|\right)>j \\
B_{r m j}=0 & \text { if } & \min \left(\left|v^{\prime}-v\right|,\left|v^{\prime}-v_{*}\right|\right)<1 / j, \\
& B_{r m j} \in C^{\infty} .
\end{array}
$$

We refer to Arkeryd and Nouri [7] for details of defining $B_{r m j}$ in such a way that the symmetry properties (2.6) are satisfied independently of $r, m$ and $j$. The sequence $\left(B_{r m j}\right)$ can be chosen so that, locally, $B_{r m j} \rightarrow B_{r m}$ as $j \rightarrow \infty$, uniformly outside sets of arbitrarily small measure in $\left(x, v, y, v_{*}\right)$.

The regularized 'gain' operator $Q_{j}^{+}(F, f)$ in (4.2) is defined as follows:

$$
Q_{j}^{+}(F, f)=\iint_{\Omega \times \mathbb{R}^{3}}\langle F\rangle_{j}\left\langle f_{*}\right\rangle_{j} \chi_{\left\{\left(v-v_{*}\right) \cdot \omega_{x y}>0\right\}} B_{r m j} d y d v_{*}
$$

where

$$
\langle F\rangle_{j}=\frac{F(x, v)}{1+F(x, v) /(j \mu(F))}
$$

and $\left\langle f_{*}\right\rangle_{j}$ is defined similarly. Notice that for every $j>0$ the operation $\langle\cdot\rangle_{j}$ is homogeneous of degree one and monotone in the sense that $f_{1} \geq f_{2}$ implies $\left\langle f_{1}\right\rangle_{j} \geq\left\langle f_{2}\right\rangle_{j}$.

Finally, we set in (4.2)

$$
\mathcal{R}_{i} g(x, v)=\left|v \cdot n_{x}\right|^{-1} \iint_{\partial \Omega^{-}} g(y, w) k_{i}(x, v, y, w)\left|w \cdot n_{y}\right| d \sigma_{y} d w,
$$


where $k_{i}$ is a sequence of mollified kernels, such that for all $i>0,\left|v \cdot n_{x}\right|^{-1} k_{i}$ are smooth and bounded, and for all $(y, w) \in \partial \Omega^{-}$and all $\varphi \in C \cap L^{\infty}\left(\partial \Omega^{+}\right)$,

$$
\iint_{\partial \Omega^{+}} k_{i}(x, v, y, w) \varphi(x, v) d \sigma_{x} d v \underset{i \rightarrow \infty}{\longrightarrow} \int_{v \cdot n_{y}>0} k(y, v, w) \varphi(y, v) d v .
$$

In addition we require that for all $i>0$ and all $(y, w) \in \partial \Omega^{-}$

$$
\iint_{\partial \Omega^{+}} k_{i}(x, v, y, w) d \sigma_{x} d v=1 \text { and } \iint_{\partial \Omega^{+}} k_{i}(x, v, y, w)|v|^{2} d \sigma_{x} d v \leq C
$$

and also that for some $\varepsilon>0$ and $0<r_{1}<r_{2}$,

$$
\iint_{\left\{v \cdot n_{x}>\varepsilon|v| ; r_{1} \leq|v| \leq r_{2}\right\}} k_{i}(x, v, y, w) d \sigma_{x} d v \geq c_{2} .
$$

All of the properties (4.4)-(4.6) are satisfied if we take $k_{i}(x, v, y, w)=\psi_{i}(x, y)$ $\varphi_{i}(v) * k(y, v, w)$, where $\varphi_{i}(v)$ is a symmetric Maxwellian with unit density, such that $\varphi_{i}(v) \rightarrow \delta(v)$, and $\psi_{i}(x, v)$ is a regularizing kernel on $\partial \Omega$ as defined in [7]. Notice also that in that case $\mathcal{R}_{i}$ satisfy (2.17), with $i$-dependent boundary Maxwellians.

To find solutions to problem (4.2) we use the classical iteration scheme in the exponential form (2.24) (in which $\alpha$ is added to the collision frequency). We set

$$
\begin{array}{rlrl}
U f^{\sharp}(x, v, s) & =\int_{0}^{s} f^{\sharp}(x, v, \tau) d \tau ; & U_{ \pm} f^{\sharp}(x, v) & =U f^{\sharp}\left(x, v, \mp s^{ \pm}\right), \\
E^{\alpha} f(x, v, s) & =\exp \left(\alpha s+U v_{n}(f)^{\sharp}\right) ; & E_{ \pm}^{\alpha} f(x, v)=E^{\alpha} f\left(x, v, \mp s^{ \pm}\right),
\end{array}
$$

and consider the sequence of iterations:

$$
\begin{aligned}
F_{0} & =0, \\
F_{n+1} & =\left(\lambda \mathcal{R}_{i} g+\alpha \mu S\right) E_{+}^{\alpha} f+U_{+}\left(\varphi_{l} * Q_{j}^{+}\left(F_{n}, f\right)^{\sharp} E^{\alpha} f\right), \\
n & =0,1,2, \ldots
\end{aligned}
$$

It is easily verified that the sequence of functions $F_{n}$ is pointwise monotone (notice the monotonicity of $Q_{j}^{+}(F, f)$ ). Using the weak form (2.28) for $F_{n}$ with $\psi=1$ and noticing that the integral of the collision term in (4.2) is nonpositive, we get

$$
\begin{aligned}
& \iint_{\partial \Omega^{-}}\left(F_{n+1}\right)_{-}\left|v \cdot n_{x}\right| d \sigma_{x} d v+\alpha \iint_{\Omega \times \mathbb{R}^{3}} F_{n} d x d v \\
\leq & \lambda \iint_{\partial \Omega^{-}} g\left|v \cdot n_{x}\right| d \sigma_{x} d v+\alpha \mu \iint_{\partial \Omega^{+}} S\left|v \cdot n_{x}\right| d \sigma_{x} d v=1 .
\end{aligned}
$$


Thus, for $\alpha>0$ fixed, the total mass of $F_{n}$ is bounded above by $1 / \alpha$, and therefore, by B. Levi's theorem, the sequence $\left(F_{n}\right)$ converges strongly in $L^{1}$. The limit function, which we denote $F$ (and which depends on $\alpha, r, m, j$ and $l$ ), can then easily be shown to satisfy (4.2) in the exponential form (cf. [7]).

We further establish upper and lower bounds for the total mass, energy and boundary fluxes of solutions $F$. First of all, passing to the limit in (4.8) we find that the total mass is bounded above by $1 / \alpha$ and the total mass outflow cannot exceed 1. Also, using the truncation for $|v|>j$ in (4.3),

$$
F(x, v)=F_{+}\left(x^{+}, v\right) \exp \left(-\int_{-s^{+}}^{0}(v(f)+\alpha)^{\sharp} d s\right), \quad \text { for } \quad|v|>j,
$$

and so,

$$
\begin{aligned}
\iint_{\Omega \times \mathbb{R}^{3}} F|v|^{2} d x d v & \leq j^{2} \iint_{\Omega \times \mathbb{R}^{3}} F d x d v+\iint_{\partial \Omega^{+}} s^{-}(x, v) F_{+}|v|^{2}\left|v \cdot n_{x}\right| d \sigma_{x} d v \\
& \leq \frac{j^{2}}{\alpha}+C(\lambda+\alpha \mu)=C_{j \alpha},
\end{aligned}
$$

where the constant $C$ depends on the diameter of $\Omega$, the constant in (4.5) and the moments of $S$. To find lower bounds, we first notice that from the exponential form (2.24),

$$
F_{-}\left(x^{-}, v\right) \geq F(x, v) \exp \left(-\int_{0}^{s^{-}}(v(f)+\alpha)^{\sharp} d s\right) .
$$

Multiplying by $|v|$ and integrating with respect to $(x, v)$ in $\Omega \times\{|v|>\delta\}$, with $\delta$ a positive constant, yields

$$
D \iint_{\partial \Omega^{-}} F_{-}(x, v)\left|v \cdot n_{x}\right| d \sigma_{x} d v \geq \delta e^{-D(m+\alpha) / \delta} \iint_{|v|>\delta} F(x, v) d x d v .
$$

In the last integral, we estimate $F$ in terms of $F_{+}$, using the exponential form (2.24) and apply the lower bound (4.6). For this take $\varepsilon>0$ as in (4.6), choose $\delta<r_{1}$ and $d=d_{\varepsilon}$ so that the cone $C_{\varepsilon, d}(x)$ defined as in (2.12) is contained in $\Omega$. We then have

$$
\begin{aligned}
& \iint_{|v|>\delta} F(x, v) d x d v \\
\geq & e^{-D(m+\alpha) / \delta} \iint_{\left\{|v|>\delta ; v \cdot n_{x^{+}}>0\right\}} S^{-}\left(x^{+}, v\right) F_{+}\left(x^{+}, v\right)\left|v \cdot n_{x^{+}}\right| d \sigma_{x^{+}} d v \\
\geq & c_{2} \lambda \frac{d}{r_{2}} e^{-D(m+\alpha) / \delta} \iint_{\partial \Omega^{-}} g\left|v \cdot n_{x}\right| d \sigma_{x} d v \\
& +\alpha \mu \frac{d}{r_{2}} \iint_{\left\{r_{1} \leq|v| \leq r_{2} ; v \cdot n_{x^{+}}>0\right\}} S\left|v \cdot n_{x^{+}}\right| d \sigma_{x^{+}} d v \\
\geq & c_{m}(\lambda+\alpha \mu)=c_{m} .
\end{aligned}
$$


This allows us to define the operator

$T:(f, g, \lambda, \mu) \mapsto\left(\frac{F}{\mu(F)}, \frac{F_{-}}{\Lambda^{-}\left(F_{-}\right)}, \frac{\Lambda^{-}\left(F_{-}\right)}{\Lambda^{-}\left(F_{-}\right)+\alpha \mu_{0}(F)}, \frac{\mu_{0}(F)}{\Lambda^{-}\left(F_{-}\right)+\alpha \mu_{0}(F)}\right)$,

where we set $\Lambda^{-}\left(F_{-}\right)=\iint_{\partial \Omega^{-}} F_{-}\left|v \cdot n_{x}\right| d \sigma_{x} d v, \mu_{0}(F)=\iint_{\Omega \times \mathbb{R}^{3}} F d x d v$, and $\mu(F)=\iint_{\Omega \times \mathbb{R}^{3}} F\left(1+|v|^{2}\right)^{\beta^{\prime} / 2} d x d v$, where $\beta^{\prime}=\max (\beta, 0)$. The operator $T$ maps the bounded convex set

$$
\begin{gathered}
\mathcal{L}=\left\{(f, g, \lambda, \mu) \in L^{1}\left(\Omega \times \mathbb{R}^{3}\right) \times L^{1}\left(\partial \Omega^{-},\left|v \cdot n_{x}\right| d \sigma_{x} d v\right) \times[0,1] \times[0,1 / \alpha]\right. \\
\left.\mid f \geq 0, g \geq 0, \mu(f)=1, \Lambda^{-}(g)=1, \lambda+\alpha \mu=1\right\}
\end{gathered}
$$

into itself. A fixed point of this operator (after a normalization so that the total mass inflow is 1) will be a solution of the following boundary-value problem:

$$
\begin{aligned}
v \cdot \nabla_{x} F+\alpha F & =\frac{1}{\mu(F)}\left(\varphi_{l} * Q_{j}^{+}(F, F)-v_{j}(F) F\right), \quad(x, v) \in \Omega \times \mathbb{R}^{3}, \\
F_{+} & =\frac{\mathcal{R}_{i} F_{-}+\alpha \mu_{0}(F) S}{\Lambda^{-}\left(F_{-}\right)+\alpha \mu_{0}(F)}, \quad(x, v) \in \partial \Omega^{+},
\end{aligned}
$$

which will be equivalent to (3.12) (with the $r$ and $m$ truncations) after we take the limits $j \rightarrow \infty$ and $i \rightarrow \infty$.

To prove that $T$ indeed has a fixed point in $\mathcal{L}$ we shall apply Schauder's principle which requires us to verify the continuity and compactness of $T$. The continuity can be checked by arguing along the lines of [7], using the monotonicity of $Q_{j}^{+}(F, f)$ and the $r$ and $m$ truncations. The compactness argument, using the $j$ truncation and the convolution with $\varphi_{l}$, can also be adapted from [7] with minimal changes. We notice also that the fixed point solutions will satisfy the upper and lower bounds for the mass, energy and boundary fluxes established above. We can further use the regularizing properties of the iterated 'gain' term as in [7] and establish that the family of the fixed point solutions $F$ corresponding to $l \rightarrow \infty$ is compact in $L^{1}\left(\Omega \times \mathbb{R}^{3}\right)$ (for this we use the smoothness and truncations in (4.3)). We can then pass to the limit $l \rightarrow \infty$ in (4.11) and remove the convolution with $\varphi_{l}$.

It remains to pass to the limit as $j \rightarrow \infty$ and then as $i \rightarrow \infty$ to recover the collision operator $Q_{r m}$ containing only the $r$ and $m$ truncations and the original boundary operator $\mathcal{R}$. We first establish a new bound for the total energy, which is uniform in $i$ and $j$. Indeed, the collision term in (4.11) satisfies

$$
\begin{aligned}
& \iint_{\Omega \times \mathbb{R}^{3}}\left(Q_{j}^{+}(F, F)-v_{j}(F) F\right)|v|^{2} d x d v \\
= & \iint_{\left(\Omega \times \mathbb{R}^{3}\right)^{2}}\left(F^{\prime} F_{*}^{\prime}-\left\langle F^{\prime}\right\rangle_{j}\left\langle F_{*}^{\prime}\right\rangle_{j}\right)|v|^{2} \chi_{\left\{\left(v-v_{*}\right) \cdot \omega_{x y}>0\right\}} B d y d v_{*} d x d v \leq 0 .
\end{aligned}
$$


Using the weak form (2.28) with $\psi=|v|^{2}$ we then obtain

$$
\begin{aligned}
\iint_{\partial \Omega^{-}} F_{-}|v|^{2}\left|v \cdot n_{x}\right| d \sigma_{x} d v & +\alpha \iint_{\Omega \times \mathbb{R}^{3}} F|v|^{2} d x d v \\
\leq & \iint_{\partial \Omega^{+}} F_{+}|v|^{2}\left|v \cdot n_{x}\right| d \sigma_{x} d v .
\end{aligned}
$$

Expressing $F_{+}$using the boundary condition in (4.11) we find that the righthand side of (4.12) can be estimated in terms of the constant in (4.5) and the second moment of $S$, which in view of (4.12) implies an $\alpha$-dependent bound for the total energy.

We further study the truncated version of the equation for the entropy, for which we multiply equation (4.11) by $\log \langle F\rangle_{j}$, integrate, and use (3.8) to get

$$
\begin{aligned}
& \quad \frac{1}{\mu(F)} \iint_{\Omega \times \mathbb{R}^{3}} e\left(\langle F\rangle_{j},\langle F\rangle_{j}\right) d x d v+\alpha \iint_{\Omega \times \mathbb{R}^{3}} F \log \langle F\rangle_{j} d x d v \\
& \leq \frac{1}{\mu(F)} \iint_{\Omega \times \mathbb{R}^{3}}\left(\varepsilon_{j}(F, F)+q_{j}(F, F)\right) d x d v \\
& \quad+\iint_{\partial \Omega^{+}} F_{+} \log \left\langle F_{+}\right\rangle_{j}\left|v \cdot n_{x}\right| d \sigma_{x} d v \\
& \quad+\iint_{\left\{\partial \Omega^{-}:\left\langle F_{-}\right\rangle_{j}<1\right\}} F_{-}\left|\log \left\langle F_{-}\right\rangle_{j}\right|\left|v \cdot n_{x}\right| d \sigma_{x} d v .
\end{aligned}
$$

Here, the terms $\varepsilon_{j}(F, F)$ and $q_{j}(F, F)$ appear due to the asymmetry between the 'gain' and 'loss' terms and the truncation in the logarithm term:

$$
\varepsilon_{j}(F, F)=-\iint_{\Omega \times \mathbb{R}^{3}}\left(F F_{*}-\langle F\rangle_{j}\left\langle F_{*}\right\rangle_{j}\right) \log \langle F\rangle_{j} \chi_{\left\{\left(v-v_{*}\right) \cdot \omega_{x y}<0\right\}} B d y d v_{*}
$$

and

$$
\begin{aligned}
q_{j}(F, F)= & -\frac{1}{1+F /(j \mu(F))} \iint_{\Omega \times \mathbb{R}^{3}}\left(\left\langle F^{\prime}\right\rangle_{j}\left\langle F_{*}^{\prime}\right\rangle_{j} \chi_{\left\{\left(v-v_{*}\right) \cdot \omega_{x y}>0\right\}}\right. \\
& \left.-F F_{*} \chi_{\left\{\left(v-v_{*}\right) \cdot \omega_{x y}<0\right\}}\right) B d y d v_{*} \\
\leq & \langle F\rangle_{j} \iint_{\Omega \times \mathbb{R}^{3}} F_{*} \chi_{\left\{\left(v-v_{*}\right) \cdot \omega_{x y}<0\right\}} B d y d v_{*} .
\end{aligned}
$$

Recalling that $B=B_{r m j}$ satisfies $B \leq m$, the integral of $q_{j}(F, F)$ is estimated as $m\left(\iint_{\Omega \times \mathbb{R}^{3}} F d x d v\right)^{2}$. Further, the integral of $\varepsilon_{j}(F, F)$ can be estimated as 
follows:

$$
\begin{aligned}
& -\iint_{\left(\Omega \times \mathbb{R}^{3}\right)^{2}}\left(F F_{*}-\langle F\rangle_{j}\left\langle F_{*}\right\rangle_{j}\right) \log \langle F\rangle_{j} \chi_{\left\{\left(v-v_{*}\right) \cdot \omega_{x y}<0\right\}} B d x d v d y d v_{*} \\
\leq & \iint_{\left\{\langle F\rangle_{j}<1\right\}}\left(F F_{*}-\langle F\rangle_{j}\left\langle F_{*}\right\rangle_{j}\right)\left|\log \langle F\rangle_{j}\right| \chi_{\left\{\left(v-v_{*}\right) \cdot \omega_{x y}<0\right\}} B d x d v d y d v_{*} \\
(4.15) \leq & \iint_{\left\{\langle F\rangle_{j}<1\right\}} F F_{*}\left|\log \langle F\rangle_{j}\right| \chi_{\left\{\left(v-v_{*}\right) \cdot \omega_{x y}<0\right\}} B d x d v d y d v_{*} \\
\leq & m \mu(F) \iint_{\left\{\langle F\rangle_{j}<1\right\}} F\left|\log \langle F\rangle_{j}\right| d x d v \\
\leq & C m \mu(F)\left(1+\iint_{\Omega \times \mathbb{R}^{3}} F\left(1+|v|^{2}\right) d x d v\right) .
\end{aligned}
$$

(The last inequality follows by a well-known argument, see e.g. [18, page 329].) By the mass and energy estimates the last term is bounded from above, uniformly in $j$.

To control the boundary integrals in (4.13) we notice that for every $i>0$ fixed, we have the pointwise estimate

$$
F_{+}(x, v) \leq \sup \left|v \cdot n_{x}\right|^{-1} k_{i}+\sup S \leq C_{i} .
$$

Therefore, the integral of $F_{+} \log F_{+}$appearing in (4.13) can be bounded uniformly in $j$ by a constant depending on $i$. We also have, similarly to (4.15),

$$
\begin{aligned}
& \iint_{\left\{\left\langle F_{-}\right\rangle_{j}<1\right\}} F_{-}\left|\log \left\langle F_{-}\right\rangle_{j}\right|\left|v \cdot n_{x}\right| d \sigma_{x} d v \\
\leq & C\left(1+\iint_{\partial \Omega^{-}} F_{-}\left(1+|v|^{2}\right)\left|v \cdot n_{x}\right| d \sigma_{x} d v\right),
\end{aligned}
$$

and so, the last term in (4.13) is bounded by a constant independent of $i$ and $j$.

Equation (4.13) now implies an $\alpha$-dependent bound for $\iint_{\Omega \times \mathbb{R}^{3}} F \log \langle F\rangle_{j}$ $d x d v$, which is uniform in $j$. In view of the mass and energy estimates it follows that the family of solutions $F$ corresponding to $j>0$ is weakly compact in $L^{1}$. Taking a sequence $\left(F^{j}\right)$ such that it has a weak $L^{1}$ limit, which we still denote by $F$, we can, for $\alpha, r, m$ and $i$ fixed, pass to the limit $j \rightarrow \infty$ in the weak form (2.28) similarly to [7]. It is also easy to verify that the sequence of boundary traces $F_{ \pm}^{j}$ is weakly compact in $L^{1}\left(\partial \Omega^{ \pm},\left|v \cdot n_{x}\right| d \sigma_{x} d v\right.$, and so, $F_{ \pm}^{j} \rightarrow F_{ \pm}$weakly in $L^{1}$. Passing to the limit in the boundary condition (4.11) and noticing that by the mass conservation, $\Lambda^{-}\left(F_{-}\right)+\alpha \mu_{0}(F)=1$, we obtain that the traces $F_{ \pm}$satisfy the boundary condition

$$
F_{+}=\mathcal{R}_{i} F_{-}+\alpha S \iint_{\Omega \times \mathbb{R}^{3}} F d x d v, \quad(x, v) \in \partial \Omega^{+}
$$


To pass to the limit in (4.16) we invoke Lemma 3.2 obtaining

$$
\begin{aligned}
\frac{1}{\mu(F)} \iint_{\Omega \times \mathbb{R}^{3}} e(F, F) d x d v & +\alpha \iint_{\Omega \times \mathbb{R}^{3}} F \log F d x d v \\
\leq C & \left(1+\iint_{\partial \Omega^{+}} F_{+}\left|v \cdot n_{x}\right| d \sigma_{x} d v\right) \leq C,
\end{aligned}
$$

which in particular implies that the entropy of $F$ is bounded uniformly in $i$. Therefore, the family of solutions $\left(F^{i}\right)$ is relatively weakly compact in $L^{1}\left(\Omega \times \mathbb{R}^{3}\right)$. This implies the corresponding weak compactness of the collision terms $Q_{r m}\left(F^{i}, F^{i}\right)$ (for $r$ and $m$ fixed): indeed $\nu\left(F^{i}\right) / \mu\left(F^{i}\right)$ are uniformly bounded, and one can also verify the inequality

$$
F^{\prime} F_{*}^{\prime} \leq K F F_{*}+\frac{K}{K \log K-K+1} F^{\prime} F_{*}^{\prime}\left(\frac{F F_{*}}{F^{\prime} F_{*}}-\log \frac{F F_{*}}{F^{\prime} F_{*}^{\prime}}-1\right),
$$

for every $K>1$ and all $\left(v-v_{*}\right) \cdot \omega_{x y}>0$, which then, in view of the bound (4.17) for the entropy production, implies the weak compactness of $Q_{r m}^{+}\left(F^{i}, F^{i}\right)$ (cf. [18] or [3]). One can then conclude using, for instance, (2.23) that the boundary traces $F_{ \pm}^{i}$ are weakly compact in $L^{1}\left(\partial \Omega^{ \pm},\left|v \cdot n_{x}\right| d \sigma_{x} d v\right)$. Choosing a subsequence of $F^{i}$ which has a limit point in $L^{1}\left(\Omega \times \mathbb{R}^{3}\right)$ we can pass to the limit $i \rightarrow \infty$ and identify the limits of $F_{ \pm}^{i}$ with the boundary traces of the limit solution.

We have therefore constructed solutions to (3.12), for every $\alpha>0, r>0$ and $m>0$. Our further strategy is to study the limits of these solutions as $\alpha \rightarrow 0, r \rightarrow 0$ and $m \rightarrow \infty$. In next two sections we shall establish the compactness properties of the approximating solutions which will allow us to pass to the limit in (3.12).

\section{5. - A priori lower bounds in domains with reflecting boundary}

In this section we establish certain 'pointwise' lower bounds for solutions (that hold almost everywhere in $x$, outside arbitrarily small sets in velocity), which will allow us to reach two important goals. First, as an immediate consequence of these results we obtain uniform lower bounds for the total mass, removing, in essence, the dependence on $m$ in estimate (4.10). Second, and more far-reaching application of the results will become apparent in Section 6 in context of the weak compactness analysis of approximating solutions.

Lower bounds of this type were obtained in [7], for convex domains $\Omega$, and when the integral collision frequency (2.3) is a locally bounded function (as in the 'hard' interactions case). Here we use geometric properties of domains with smooth boundary to get rid of the convexity assumption, together with a more detailed analysis of characteristics on which the collision frequency is 
bounded: this will allow us to treat a more general collision frequency case. We shall focus on the problem with boundary condition (2.14); in the case of the given inflow condition (2.20) similar lower bounds can be obtained in a much more straightforward way (see proof of Theorem 2.3).

While the main applications of our analysis concern the approximating solutions constructed in the previous section, the results themselves hold in a rather abstract setting. Therefore, we shall consider solutions of the differential inequality

$$
v \cdot \nabla_{x} f+v f \geq 0, \quad(x, v) \in \Omega \times \mathbb{R}^{3},
$$

(to be satisfied for almost all characteristics, in the sense of an integral form similar to (2.24)) which also have boundary traces satisfying the inequality

$$
f_{+} \geq \mathcal{R} f_{-}, \quad(x, v) \in \partial \Omega^{+},
$$

where $\mathcal{R}$ is as in (2.15). Here $v \geq 0$ is a measurable function on $\Omega \times \mathbb{R}^{3}$, possibly depending on $f$, which satisfies the following.

Assumption 5.1. For a certain $R>0$, and for every $\eta>0$ there is a constant $A_{\eta}>0$ (we suppress the dependence on $R$ for brevity), such that

a) for a.a. $x \in \partial \Omega$

$$
\int_{-s^{+}(x, v)}^{s^{-}(x, v)} v^{\sharp}(x, v, s) d s \leq A_{\eta},
$$

for all $v \in\left\{v \in \mathbb{R}^{3}\left|\left(v \cdot n_{x}\right)<0,\right| v \mid \leq R\right\}$ outside an $x$-dependent set of measure $\eta$ and

b) for a.a. $x \in \Omega$, condition (5.3) holds for $v \in\{|v| \leq R\}$, outside an $x$ dependent set of measure $\eta$.

It is easy to see that the above assumption is verified for $v=v(f)$ given by (2.3), in the cases of both 'hard' and 'soft' interactions, provided that $\mu(f)$ is bounded. Indeed, for $\beta>0$ in (2.9) it suffices to see that according to (2.26) we have $\sup _{x \in \Omega} v(f) \leq C_{R}$ for $|v| \leq R$ and so, for every $r_{1}>0$ condition (5.3) is satisfied for $r_{1} \leq|v| \leq R$ with $A_{\eta}=C_{R} D \kappa / r_{1}$, where $D$ is the diameter of $\Omega$. In the case of 'soft' interactions (2.27) implies

$$
\text { meas }\left\{v|| v \mid \leq R, \int v^{\sharp} d s>A\right\} \leq \text { meas }\left\{v|| v \mid \leq R, \sup _{x \in \Omega} v(x, v)>\frac{A r_{1}}{D}\right\} \leq \frac{\eta}{2},
$$

if $A_{\eta}=2 C_{R} D \kappa / \eta r_{1}$, and taking $r_{1}$ so that $B\left(0, r_{1}\right)=\eta / 2$ we obtain (5.3) for a.a. $x \in \Omega$ and for $v$ in an $x$-dependent set with complement of measure $\eta$ in $\{|v| \leq R\}$.

In the sequel, we will use the following geometric concept. 
Definition 5.1. Given $\varepsilon \in(0,1)$ we say that two points $x$ and $y$ on $\partial \Omega$ are $\varepsilon$-nontangentially connected if the open segment $(x ; y)=\{\lambda x+(1-\lambda) y \mid 0<$ $\lambda<1\}$ is contained in $\Omega$ and

$$
(y-x) \cdot n_{x}>\varepsilon|y-x| \text { and }(x-y) \cdot n_{y}>\varepsilon|y-x| .
$$

We sometimes omit $\varepsilon$ from the notation, writing 'nontangentially connected' when the value of $\varepsilon$ is clear from the context. We denote by $V_{\varepsilon}(x)$ the set of all points $y \in \partial \Omega$ nontangentially connected with $x$. We also define, for $S \subseteq \partial \Omega$

$$
V_{\varepsilon}(S)=\bigcap_{x \in S} V_{\varepsilon}(x),
$$

the set of points, nontangentially connected with $S$. If $S^{\prime} \subseteq V_{\varepsilon}(S)$, we say that $S$ and $S^{\prime}$ are nontangentially connected (notice that nontangentiality of sets so defined is a symmetric relation). The subsequent proofs will use estimates for fluxes of solutions to (5.1) on nontangentially connected parts of the boundary. For this we need the geometric fact formulated in next lemma. We denote by $\Gamma_{\delta}(x)$ the neighborhood of $x \in \partial \Omega$ obtained by intersecting $\partial \Omega$ with the open ball $B(x, \delta)$ in $\mathbb{R}^{3}$.

LEMMA 5.1. Let $\Omega$ be a bounded domain with $C^{1}$ boundary satisfying the cone condition (2.12). There exist constants $N>0, \varepsilon>0$ and $\delta>0$ such that any two points $x, y$ on $\partial \Omega$ can be connected by a polygonal path in $\bar{\Omega}$ with nodes $\left\{x_{i}\right\}_{i=1}^{N}$ on $\partial \Omega$, such that $x_{1}=x, x_{N}=y$, and $\delta$-neighborhoods $\Gamma_{\delta}\left(x_{i}\right)$ and $\Gamma_{\delta}\left(x_{i+1}\right)$ are $\varepsilon$-nontangentially connected for $i=1, \ldots, N-1$.

Proof. First, we show that $\varepsilon>0$ can be chosen so that for all $x \in \partial \Omega$, the set $V_{4 \varepsilon}(x)$ is nonempty. For this we use the cone condition, taking $\theta$ and $d=d_{\theta}$ according to (2.12). Let $\Sigma_{\theta}(x)$ denote the solid angle of directions $\omega_{x y}$ corresponding to the cone $C_{\theta, d}$. Integrating over $\omega_{x y}$ in $\Sigma_{\theta}(x)$, we can estimate the area of the part of $\partial \Omega$ 'visible' in this solid angle. Thus,

$$
|\partial \Omega| \geq \int_{\Sigma_{\theta}(x)} \frac{|y-x|^{3} d \omega_{x y}}{(x-y) \cdot n_{y}} \geq d^{2} \int_{\Sigma_{\theta}(x)} \frac{|x-y| d \omega_{x y}}{(x-y) \cdot n_{y}} .
$$

By Chebyshev's inequality applied to the last integral, the inequality $(x-y) \cdot n_{y} \leq$ $\eta|x-y|$ can only hold for $\omega_{x y}$ from a set of area $\eta|\partial \Omega| / d^{2}$ in $\Sigma_{\theta}(x)$. Choosing $\eta$ small enough, we can guarantee that for all $x$ this set has nonempty complement in $\Sigma_{\theta}(x)$. Taking $4 \varepsilon=\min (\theta, \eta)$, it follows that $V_{4 \varepsilon}(x)$ is nonempty for all $x$.

Next, we extend the nontangentiality conditions to $\delta$-neighborhoods in the following way. We first show that if $\delta$ is chosen small enough, then for all $x \in \partial \Omega$ there exists $y \in \partial \Omega$ such that $\Gamma_{\delta}(x)$ and $\Gamma_{\delta}(y)$ are $2 \varepsilon$-nontangentially connected. To prove this take first $\delta<d / 4$ where $d=d_{4 \varepsilon}$ is determined 
by (2.12) with $C_{4 \varepsilon, d}(x)$ as the cone. Picking $y \in V_{4 \varepsilon}(x)$, we have for every $x^{\prime} \in \Gamma_{\delta}(x)$ and $y^{\prime} \in \Gamma_{\delta}(y)$, the inequality $\left|x^{\prime}-y^{\prime}\right|>d / 2$, and moreover,

$$
\begin{aligned}
\left(x^{\prime}-y^{\prime}, n_{y^{\prime}}\right) & =\left(x-y, n_{y}\right)+\left(y-y^{\prime}, n_{y}\right)+\left(x^{\prime}-x, n_{y^{\prime}}\right)+\left(x-y^{\prime}, n_{y^{\prime}}-n_{y}\right) \\
& \geq 4 \varepsilon|x-y|-2 \delta-\varepsilon\left|x-y^{\prime}\right| \geq 3 \varepsilon\left|x^{\prime}-y^{\prime}\right|-(9 \varepsilon+2) \delta \\
& \geq 3 \varepsilon\left|x^{\prime}-y^{\prime}\right|-\frac{(9 \varepsilon+2) \delta}{2 d}\left|x^{\prime}-y^{\prime}\right|>2 \varepsilon\left|x^{\prime}-y^{\prime}\right|,
\end{aligned}
$$

provided $\delta$ is small enough, so that $\left|n_{y^{\prime}}-n_{y}\right|<\varepsilon$ and $\delta<2 \varepsilon d /(9 \varepsilon+2)$. A similar calculation shows that

$$
\left(y^{\prime}-x^{\prime}, n_{x^{\prime}}\right)>2 \varepsilon\left|x^{\prime}-y^{\prime}\right|,
$$

for sufficiently small $\delta$. Thus, we have shown that $\Gamma_{\delta}(x)$ and $\Gamma_{\delta}(y)$ are nontangentially connected. Since $n_{x}$ is a uniformly continuous function on $\partial \Omega$, the constant $\delta$ can be chosen uniformly for all $x \in \partial \Omega$. Now taking an arbitrary $x^{\prime} \in \Gamma_{\delta}(x)$ we can repeat the above argument and by choosing $\delta$ small enough, prove that also $\Gamma_{\delta}\left(x^{\prime}\right)$ is $\varepsilon$-nontangentially connected with $\Gamma_{\delta}(y)$.

The sequence of nodes $\left(x_{i}\right)_{i=1}^{N}$ can now be constructed in the following way. Take a covering of $\partial \Omega$ by balls of radius $\delta$ so that for a subsequence of their centers, $\left(x_{i}^{\prime}\right)_{i=1}^{M}$, it holds $x_{1}^{\prime}=x, x_{M}^{\prime}=y$ and $x_{i+1}^{\prime} \in \Gamma_{\delta}\left(x_{i}^{\prime}\right)$ for $i=1, \ldots, M$. Let $x_{1}=x$ and take $x_{2} \in V_{4 \varepsilon}\left(x_{1}\right)$ so that $\Gamma_{\delta}\left(x_{2}\right)$ and $\Gamma_{\delta}\left(x_{1}\right)$ are $2 \varepsilon$-nontangentially connected. Let $x_{3}=x_{2}^{\prime}$. Since $x_{3} \in \Gamma_{\delta}\left(x_{1}\right)$, it follows by the previous argument that $\Gamma_{\delta}\left(x_{3}\right)$ is $\varepsilon$-nontangentially connected with $\Gamma_{\delta}\left(x_{2}\right)$. Defining $x_{2 i-1}=x_{i}^{\prime}$, we can continue the process, taking on each step $x_{i}^{\prime}$ as a new starting point and finding $x_{2 i}$ so that $\Gamma_{\delta}\left(x_{2 i}\right)$ is $2 \varepsilon$-nontangentially connected with $\Gamma_{\delta}\left(x_{i}^{\prime}\right)$ and $\varepsilon$-nontangentially connected with $\Gamma_{\delta}\left(x_{i+1}^{\prime}\right)$. After $M-1$ steps we end up with a sequence of $N=2 M-1$ points $\left(x_{i}\right)_{i=1}^{N}$, which satisfies the requirements of the lemma.

In next lemma we establish a uniform in $x \in \partial \Omega$ lower estimate for the density of the mass outflow

$$
\rho(x)=\int_{v \cdot n_{x}<0} f_{-}(x, v)\left|v \cdot n_{x}\right| d v,
$$

generalizing the result of Lemma 2.3 in [7].

LEMMA 5.2. Let $\Omega$ be a bounded domain with $C^{1}$ boundary satisfying the cone condition (2.12), and let $f$ satisfy (5.1), (5.2), with the function $v$ satisfying Assumption 5.1. Then, there are constants $\eta>0, b>0$ and $c>0$, such that

$$
\rho(x) \geq b e^{-c A} \int_{\partial \Omega} \rho d \sigma_{x},
$$

where $A=A_{\eta}$ from (5.3). Here the constants $b, c$ and $\eta$ depend only on $R$, the geometry of the domain $\Omega$ and the lower bound (2.19) for the kernel $k$ in (2.15). 
Proof. The proof is based on two facts. First, $f_{-}(x, v)$ can be bounded from below using (2.25):

$$
f_{-}(x, v) \geq e^{-A} \rho\left(x^{+}(x, v)\right) \Phi_{1}(|v|),
$$

on those characteristics for which condition (5.3) is fulfilled. We further fix the constants $0<r_{1}<r_{2} \leq R$, take $\varepsilon>0$ as in the previous lemma, integrate the above inequality with respect to $v$ in $\left\{r_{1} \leq|v| \leq r_{2}\right\}$ and use the change of variables $v \mapsto\left(|v|, x^{+}(x, v)\right)$ :

$$
\begin{aligned}
\rho(x) & \geq e^{-A} \int_{\left\{v \mid v \cdot n_{x}<0 ; \int v^{\sharp} d s \leq A\right\}} d v\left|v \cdot n_{x}\right| \rho\left(x^{+}(x, v)\right) \Phi_{1}(|v|) \\
& \geq e^{-A} \int_{r_{1}}^{r_{2}} d|v| \Phi_{1}(|v|)|v|^{3} \int_{\left\{x^{+} \in V_{\varepsilon}(x) \mid \int v^{\sharp} d s \leq A\right\}} d \sigma_{x}+K\left(x, x^{+}\right) \rho\left(x^{+}\right) .
\end{aligned}
$$

Here,

$$
K\left(x, x^{+}\right)=\frac{\left|\left(x-x^{+}\right) \cdot n_{x}\right|\left|\left(x-x^{+}\right) \cdot n_{x}\right|}{\left|x-x^{+}\right|^{4}} \geq c_{\varepsilon} \text { for } \quad x^{+} \in V_{\varepsilon}(x),
$$

the last inequality following directly from (5.4). Thus, $\rho(x)$ can be estimated pointwise by the total flux through the part of the boundary which is $\varepsilon$-nontangentially connected with $x$ :

$$
\rho(x) \geq c_{3} e^{-A} \int_{r_{1}}^{r_{2}} d|v| \int_{\left\{x^{+} \in V_{\varepsilon}(x) \mid \int v^{\sharp} d s \leq A\right\}} d \sigma_{x}+\rho\left(x^{+}\right),
$$

where $c_{3}=c_{\varepsilon} \min _{r_{1} \leq|v| \leq r_{2}} \Phi_{1}(|v|)|v|^{3}$.

Another basic estimate will allow us to compare fluxes through different parts of the boundary. Let $S$ and $S^{\prime}$ be two $\varepsilon$-nontangentially connected sets in $\partial \Omega$. Using estimate (5.6) and the lower bound (2.19) for the boundary kernel we obtain:

$$
\begin{aligned}
& \int_{S^{\prime}} \rho\left(x^{-}\right) d \sigma_{x^{-}} \\
\geq & e^{-A} \int_{S^{\prime}} d \sigma_{x^{-}} \int_{\left\{x^{+} \in S ; \int \nu^{\sharp} d s \leq A\right\}} d v\left|v \cdot n_{x^{-}}\right| \rho\left(x^{+}\left(x^{-}, v\right)\right) \Phi_{1}(|v|) \\
= & e^{-A} \int_{S} d \sigma_{x^{+}} \rho\left(x^{+}\right) \int_{\left\{x^{-} \in S^{\prime} ; \int v^{\sharp} d s \leq A\right\}} d v\left|v \cdot n_{x^{+}}\right| \Phi_{1}(|v|) \\
\geq & c_{4} e^{-A} \int_{S} \rho\left(x^{+}\right) d \sigma_{x^{+}} .
\end{aligned}
$$

Here,

$$
c_{4}=\varepsilon \min _{x^{+} \in S} \int_{\left\{x^{-} \in S^{\prime} ; \int v^{\sharp} d s \leq A\right\}} \Phi_{1}(|v|)|v| d v
$$


is positive as long as the area $\left|S^{\prime}\right|$ is bounded away from zero. Indeed, since $S$ and $S^{\prime}$ are nontangentially connected, for all $x^{+} \in S$ the solid angle of directions of $v$ such that $x^{-}\left(x^{+}, v\right) \in S^{\prime}$, is uniformly bounded from below. The set of integration in $v$ will then have the volume bounded below, if we choose the constant $\eta$ in the definition of $A$ small enough.

Now take constants $\varepsilon$ and $\delta$ as in the previous lemma and cover $\partial \Omega$ by $n$ balls of radius $\delta$. For one of the balls, say $B\left(x^{*}, \delta\right)$, we have, obviously,

$$
\int_{\Gamma_{\delta}\left(x^{*}\right)} \rho(x) d \sigma_{x} \geq \frac{1}{n} \int_{\partial \Omega} \rho(x) d \sigma_{x} .
$$

Taking $x \in \partial \Omega$ and arguing as in the proof of Lemma 5.1, we choose $y$ so that $\Gamma_{\delta}(y)$ is $\varepsilon$-nontangentially connected with $\Gamma_{\delta}(x)$. By (5.7) and (5.8) it follows then,

$$
\begin{aligned}
\rho(x) & \geq c_{3} e^{-A} \int_{r_{1}}^{r_{2}} d|v| \int_{\left\{y^{\prime} \in \Gamma_{\delta}(y) \mid \int \nu^{\sharp} d s \leq A\right\}} \rho\left(y^{\prime}\right) d \sigma_{y^{\prime}} \\
& \geq c_{5} e^{-2 A} \int_{\Gamma_{\delta}(x)} \rho\left(x^{\prime}\right) d \sigma_{x^{\prime}},
\end{aligned}
$$

where $c_{5}$ depends on $\varepsilon, \delta, r_{1}$ and $r_{2}$ and is uniformly bounded away from zero for $x \in \partial \Omega$. Using Lemma 5.1 take the polygonal path with the nodes $\left\{x_{i}\right\}_{i=1}^{N}$, connecting $x$ and $x^{*}$. Since the consequent nodes of the path are $\varepsilon$-nontangentially connected, it follows by applying inequality (5.8),

$$
\int_{\Gamma_{\delta}\left(x_{i+1}\right)} \rho(x) d \sigma_{x} \geq c_{4}(\varepsilon, \delta) e^{-A} \int_{\Gamma_{\delta}\left(x_{i}\right)} \rho(x) d \sigma_{x}
$$

By repeated application of this inequality we obtain using (5.9),

$$
\rho(x) \geq c(\varepsilon, \delta) e^{-(N+1) A} \int_{\Gamma_{\delta}\left(x^{*}\right)} \rho(x) d \sigma_{x} \geq \frac{1}{n} c(\varepsilon, \delta) e^{-(N+1) A} \int_{\partial \Omega} \rho(x) d \sigma_{x},
$$

which proves the lemma.

We conclude this section by an application of the method to solutions of the boundary-value problem (3.3), (3.4), obtaining lower bounds for solutions inside the domain that hold 'pointwise' in the $x$ variable. This property depends crucially on the part b) of Assumption 5.1 (which indeed has not been used so far). In view of the normalization condition (3.13) it is more convenient to express the bounds in terms of the total mass inflow (rather than the outflow, as in Lemma 5.2). 
LEMMA 5.3. For $\alpha \geq 0$ let $f$ be a solution of equation (3.3), with finite weighted mass $\mu(f)$ as in (3.11), which also satisfies the boundary condition (3.4) with the source term (3.5). For every $\varepsilon>0$ and for every $R>0$ there exists a constant $c$ depending on $\varepsilon, R$, and $\kappa$ in (3.11), in addition to the conditions of Lemma 5.2, such that

$$
f(x, v) \geq c \iint_{\partial \Omega^{+}} f_{+}\left|v \cdot n_{x}\right| d \sigma_{x} d v,
$$

for a.a. $x \in \Omega$ and for $v$ in an $x$-dependent set with complement of measure $\varepsilon$ in $\{|v| \leq R\}$.

Proof. Fixing $R>0$ and $\varepsilon>0$ we pick the set of $v$ in $\{|v| \leq R\}$, with complement of measure $\varepsilon$, for which Assumption 5.1 is verified. The exponential estimate (2.25) then implies

$$
f(x, v) \geq e^{-A} \rho\left(x^{+}(x, v)\right) \Phi_{1}(|v|) \geq c \iint_{\partial \Omega^{-}} f_{-}\left|v \cdot n_{x}\right| d \sigma_{x} d v,
$$

for a.a. $x \in \Omega$ and for $v$ from the above set, where we estimated the Maxwellian $\Phi_{1}(|v|)$ from below by a positive constant for $|v| \leq R$ and used the bound of Lemma 5.2. We then use (2.25) for $x \in \partial \Omega$ to get

$$
f_{-}(x, v) \geq e^{-A} f_{+}\left(x^{+}(x, v), v\right)
$$

and perform the change of variables $\left(v, x^{-}\right) \mapsto\left(v, x^{+}\right)$in the integral (which preserves the boundary measure $\left.\left|v \cdot n_{x}\right| d \sigma_{x} d v\right)$ to obtain

$$
\begin{aligned}
& \iint_{\partial \Omega^{-}} f_{-}\left(x^{-}, v\right)\left|v \cdot n_{x^{-}}\right| d \sigma_{x^{-}} d v \\
\geq & e^{-A} \iint_{\left\{v \cdot n_{x^{+}}>0, \int v(f)^{\sharp} d s \leq A\right\}} f_{+}\left(x^{+}, v\right)\left|v \cdot n_{x^{+}}\right| d \sigma_{x^{+}} d v
\end{aligned}
$$

and notice that for a.a. $x \in \partial \Omega$ the set of integration in $v$ has complement of measure at most $\varepsilon$ in $\left\{v\left|v \cdot n_{x}<0,\right| v \mid \leq R\right\}$. We further estimate

$$
f_{+}\left(x^{+}, v\right) \geq \rho\left(x^{+}\right) \Phi_{1}(|v|)+\alpha S\left(x^{+}, v\right) \iint_{\Omega \times \mathbb{R}^{3}} f d x d v
$$

and recall that $\Phi_{1}(|v|)$ and $S\left(x^{+}, v\right)$ are Maxwellians with densities and temperatures uniformly bounded from below. This implies

$$
\begin{aligned}
& \iint_{\partial \Omega^{-}} f_{-}\left|v \cdot n_{x}\right| d \sigma_{x} d v \\
\geq & c\left(\iint_{\partial \Omega^{-}} f_{-}\left|v \cdot n_{x}\right| d \sigma_{x} d v+\alpha \iint_{\Omega \times \mathbb{R}^{3}} f d x d v\right) \\
= & c \iint_{\partial \Omega^{+}} f_{+}\left|v \cdot n_{x}\right| d \sigma_{x} d v,
\end{aligned}
$$

where $c$ is an absolute constant. This completes the proof of the lemma. 


\section{6. - Weak compactness of approximating solutions}

The aim of this section is to prove that the family of the approximating solutions $(F)=\left(F^{\alpha r m}\right)$ (and the corresponding family $(f)=\left(f^{\alpha r m}\right)$ ) constructed in Section 4 is relatively compact in the weak topology of $L^{1}\left(\Omega \times \mathbb{R}^{3}\right)$. The symmetries of the truncated collision operator allow us to use the a priori bounds for the energy and the entropy production of solutions established in Section 3. Therefore, our next step will be to establish uniform bounds for the total mass $\iint_{\Omega \times \mathbb{R}^{3}} F d x d v$, based on the above a priori estimates. This result is formulated below as Lemma 6.3. The key argument, presented in Lemmas 6.1 and 6.2 uses the structure of the entropy production functional, following the approach in [5], [6], [7], [8]. As a corollary of the entropy production argument we also obtain a certain condition of equi-integrability in $v$ in Lemma 6.4. We next use the properties of the transport operator in Lemmas 6.5 and 6.6 to show that the equi-integrability also holds with respect to the $x$ variable. This will complete the proof of weak compactness.

We shall start by adapting the bounds of Section 3 and 5 to solutions of the auxiliary problem (3.12). Recalling that solutions $F=F^{\alpha r m}$ satisfy the conditions of the unit mass inflow (3.13) and using Lemma 3.1 we obtain the bound

$$
\iint_{\Omega \times \mathbb{R}^{3}} F|v|^{2} d x d v \leq C_{1}
$$

where $C_{1}$ is an absolute constant. Further, to apply the estimate of Lemma 3.2 we use a standard argument (cf. (4.15)) to find

$$
-\alpha \iint_{\Omega \times \mathbb{R}^{3}} F \log F d x d v \leq C \alpha\left(1+\iint_{\Omega \times \mathbb{R}^{3}} F\left(1+|v|^{2}\right) d x d v\right) .
$$

With the total energy bounded according to (6.1) and using an obvious bound by $1 / \alpha$ for the total mass of $F$, we get that the right-hand side of (6.2) is bounded by an absolute constant. Further, the source term $g(x, v)=\alpha S(x, v) \iint_{\Omega \times \mathbb{R}^{3}} F$ $d x d v$ in the boundary condition (3.12) is bounded uniformly by an absolute constant and has all $v$-moments bounded. Therefore, we obtain the inequality

$$
\iint_{\Omega \times \mathbb{R}^{3}} e(F, F) d x d v \leq C_{2} \iint_{\Omega \times \mathbb{R}^{3}} F\left(1+|v|^{2}\right) d x d v
$$

where $C_{2}$ is an absolute constant, and we augmented the moment of order $\beta^{\prime}=\max (0, \beta)$ to a moment of order 2 .

It is also easy to adapt the bounds provided by Lemma 5.3 to solutions of the auxiliary problem. For this recall that the integral collision frequency in (3.12) is multiplied by the factor $1 / \mu(F)$. Therefore, the bounds (2.26) and (2.27) become expressed in terms of absolute constants, and we can repeat the arguments of Lemma 5.3 to obtain lower bounds for $F$ which are uniform 
with respect to $\alpha, r$ and $m$. In particular, this implies that the total weighted mass $\mu(F)$ is bounded below uniformly with respect to our parameters.

The rest of the argument is as follows: assuming that solutions $F$ 'develop a concentration' in a certain region of the $(x, v)$-space, we compare their values with those of the integrand of the entropy production functional, establishing that, 'pointwise' in a certain subset of $(x, v)$ and in a 'suitably large' set of $\left(y, v_{*}\right)$,

$$
F F_{*}\left(\frac{F^{\prime} F_{*}^{\prime}}{F F_{*}}-\log \frac{F^{\prime} F_{*}^{\prime}}{F F_{*}}-1\right) B \geq c F,
$$

where the constant $c$ includes lower bounds for $B, F_{*}$ and the expression in parentheses (the latter is a convex function of the quotient $F^{\prime} F_{*}^{\prime} / F F_{*}$ and tends to infinity as this quotient approaches zero). In particular, we will look for regions in the space of all variables such that $F_{*}$ and $B$ are bounded from below, the quotient $F_{*}^{\prime} / F_{*}$ is of order 1 , and $F^{\prime} / F \ll 1$. We will then find, arguing by contradiction, that the total mass of $F$ is uniformly bounded, and will also establish an equi-integrability condition for sets with small $v$-sections. We start with a technical lemma required to establish a condition of the type (6.4).

Lemma 6.1. Let $\mu>0$ and let $S$ be a bounded measurable subset of $\Omega \times \mathbb{R}^{3}$, for which $\iint_{S} F(x, v) d x d v=\mu$. Then there exist constants $c_{6}, c_{7}, c_{8}, c_{9}$ and $c_{10}$, a set $S^{\prime} \subseteq \Omega \times \mathbb{R}^{3}$ for which $\iint_{S^{\prime}} F(x, v) d x d v=\mu / 2$, and for every $(x, v) \in S^{\prime}$, there exists a set $P_{x v} \subseteq \Omega \times \mathbb{R}^{3}$, with $\left|P_{x v}\right|>c_{10}$, and such that

$$
\begin{aligned}
B\left(x, v, y, v_{*}\right) & \geq c_{9}>0, \\
F\left(x, v^{\prime}\right) & \leq c_{8} F(x, v)\left|S_{x}\right| / \mu, \\
0<c_{7} & \leq F\left(y, v_{*}\right), \\
F\left(y, v_{*}^{\prime}\right) & \leq c_{6},
\end{aligned}
$$

for $(x, v) \in S^{\prime}$ and for $\left(y, v_{*}\right) \in P_{x v}$, where $S_{x}=\left\{v \in \mathbb{R}^{3} \mid(x, v) \in S\right\}$ is the $v$-section of $S$ at $x$. The sets $S^{\prime}$ and $P_{x v}$ may depend on the choice of $F$ from the family of solutions $\left(F^{\alpha r m}\right)$, but the values of the constants are uniform with respect to $\alpha, r$ and $m$.

Proof. We fix $R>0$ so that $S \subseteq \Omega \times\{|v| \leq R / 2\}$. To establish the first of the inequalities (6.5) we shall look for lower bounds of the factors $b(x, y)$, $h(z)$ and $\left|v-v_{*}\right|^{\beta}$ in (2.7), (2.9). First, we fix $0<\theta<1$ and show that for $\eta$ suitably small and for every $x \in \Omega$ the cone

$$
\left\{x^{\prime}|| x^{\prime}-x\left|\leq \eta,\left(x^{\prime}-x\right) \cdot n_{y}>\theta\right| x^{\prime}-x \mid\right\},
$$

for certain $y \in \partial \Omega$ is contained in $\Omega$. Indeed, take $\eta=\min \left(c_{2}, d / 2\right)$, where $c_{2}$ is given by (2.8) and $d=d_{\theta}$ is chosen according to (2.12). If $\operatorname{dist}(x, \partial \Omega)>\eta$, the statement is evidently true. In the other case we may take $y$ for which

$$
\operatorname{dist}(x, y)=\inf _{y \in \partial \Omega} \operatorname{dist}(x, y)
$$


(the infimum is attained since $\partial \Omega$ is smooth). It is easily verified that for such $y$ we must have $(x-y) \cdot n_{y}=0$. Using (2.12) we then see that the cone (6.6) is contained in $\Omega$. The lower bound (2.8) then implies the following: for every $x \in \Omega$ the function $b(x, y)$ is bounded away from zero for $y$ in a set of measure $c_{11}$, where $c_{11}$ is the measure of the cone (6.6).

We next show that for every $(x, v) \in S$ and for every $\omega_{x y}$ corresponding to $y$ in the cone (6.6) there is a sufficiently large set of $v_{*}$ such that the product

$$
h(\cos \vartheta)\left|v-v_{*}\right|^{\beta},
$$

where $\cos \vartheta=\left(\left(v-v_{*}\right) \cdot \omega_{x y}\right) /\left|v-v_{*}\right|$, is positively bounded from below. By 'sufficiently large' here we mean that for every $\varepsilon_{1}>0$ we can find a set of $v_{*}$ in

$$
B_{R+}(v)=\left\{v_{*} \in \mathbb{R}^{3}|| v_{*} \mid \leq R,\left(v-v_{*}\right) \cdot \omega_{x y}<0\right\}
$$

with complement of measure $\varepsilon_{1}$. Indeed, given $\varepsilon_{1}>0$, we see that the conditions $\left|v_{*}\right| \leq R$ and $|v| \leq \frac{R}{2}$ imply that $\left|v-v_{*}\right| \leq \frac{3 R}{2}$. Taking $\rho>0$ suitably small (depending on $\varepsilon_{1}$ ) we exclude $v_{*}$ for which $\left|v-v_{*}\right|>\rho$, which implies a bound from below for the factor $\left|v-v_{*}\right|^{\beta}$. Next, choosing $\varepsilon$ in (2.10) suitably small (depending on $\varepsilon_{1}$ ) we take a constant $c_{\varepsilon}>0$ and a set of $\vartheta$ with complement of measure $\varepsilon$ in $\left(0, \frac{\pi}{2}\right)$ for which $h(\cos \vartheta)>c_{\varepsilon}$. This implies that for every $\varepsilon_{1}>0$ the sets

$$
\left\{v_{*} \in \mathbb{R}^{3}|| v_{*}|\leq R,| v-v_{*} \mid \geq \rho,\left(v-v_{*}\right) \cdot \omega_{x y}<0, h(\cos \vartheta)>c_{\varepsilon}\right\},
$$

which deliver $\varepsilon_{1}$-dependent lower bounds for the product (6.7), occupy the part of the set (6.8) with complement of measure $\varepsilon_{1}$.

Further, to satisfy the second inequality (6.5) we shall choose a subset $S^{\prime}$ of $S$ with the properties specified below. First we take a measurable set $\Omega^{\prime} \subseteq \Omega$, such that for every $x \in \Omega^{\prime}$

$$
\int_{S_{x}} F(x, v) d v \geq \frac{\mu \delta^{2}}{4 C_{1}} \int_{|v| \geq \delta} F(x, v) d v
$$

where $C_{1}$ is the constant in (6.1) and $S_{x}=\left\{v \in \mathbb{R}^{3} \mid(x, v) \in S\right\}$. Denoting by $S_{0}^{\prime}$ the set $\left\{(x, v) \in S \mid x \in \Omega^{\prime}\right\}$, we see that $S_{0}^{\prime}$ carries at least $3 / 4$ of the mass $\mu$, since

$$
\iint_{S \backslash S_{0}^{\prime}} F(x, v) d x d v=\int_{\Omega \backslash \Omega^{\prime}} \int_{S_{x}} F(x, v) d x d v \leq \frac{\mu \delta^{2}}{4 C_{1}} \iint_{|v| \geq \delta} F(x, v) d x d v \leq \frac{\mu}{4} .
$$

Now we define the set $S^{\prime}$ by taking for every $x \in \Omega^{\prime}$ the values of $v$ for which $F(x, v)$ is at least $1 / 4$ of its mean value over $S_{x}$ :

$$
S^{\prime}=\left\{(x, v) \in S_{0}^{\prime} \mid F(x, v) \geq \frac{1}{4} \frac{1}{\left|S_{x}\right|} \int_{S_{x}} F(x, v) d v\right\} .
$$


This set is non-empty, since

$$
\begin{aligned}
\iint_{S_{0}^{\prime} \backslash S^{\prime}} F(x, v) d x d v & =\iint_{S_{0}^{\prime}} F(x, v) \chi_{\left\{F<\frac{1}{4} \frac{1}{\left|S_{x}\right|} \int_{S_{x}} F(x, v) d v\right\}} d x d v \\
& \leq \frac{1}{4} \int_{\Omega^{\prime}} d x \int_{S_{x}} d v \frac{1}{\left|S_{x}\right|} \int_{S_{x}} F(x, w) d w \leq \frac{\mu}{4} .
\end{aligned}
$$

It follows also that $S^{\prime}$ carries at least half of the mass $\mu$.

We next see that for every $\varepsilon_{2}>0$ the second inequality (6.5) holds, with $c_{8}$ dependent on $\varepsilon_{2}$, for $\left|v^{\prime}\right| \leq R$ outside a set of measure $\varepsilon_{2}$. Indeed, by Chebyshev's inequality, for every $x \in \Omega$ and every $\eta>0$

$$
\text { meas }\left\{v^{\prime}|| v^{\prime} \mid \geq \delta, F\left(x, v^{\prime}\right)>\frac{1}{\eta} \int_{|v| \geq \delta} F(x, v) d v\right\} \leq \eta .
$$

Thus, for every $\eta>0$, combining (6.9), (6.10) and (6.11), we can verify for $(x, v) \in S^{\prime}$ and for $\left|v^{\prime}\right| \geq \delta$, outside an $x$-dependent set of volume $\eta$, the following chain of inequalities:

$$
\left|S_{x}\right| F(x, v) \geq \frac{1}{4} \int_{S_{x}} F(x, v) d v \geq \frac{\mu \delta^{2}}{16 C_{1}} \int_{|v| \geq \delta} F(x, v) d v \geq \frac{\mu \eta \delta^{2}}{16 C_{1}} F\left(x, v^{\prime}\right) .
$$

This implies that given $\varepsilon_{2}>0$, and choosing $\delta>0$ and $\eta>0$ so that $|B(0, \delta)|+\eta<\varepsilon_{2}$, the second estimate in (6.5) is valid with $c_{8}=16 C_{1} / \eta \delta^{2}$, for $(x, v) \in S^{\prime}$ and for $\left|v^{\prime}\right| \leq R$, outside an $x$-dependent set of measure $\varepsilon_{2}$.

Let us denote by $\Delta_{x}$ the above set of measure $\varepsilon_{2}$. We then show that given $\varepsilon_{3}>0$ and $(x, v) \in S^{\prime}$, by choosing $\varepsilon_{2}$ small enough, we can find a set of $y$ in the cone (6.6) with measure bounded away from zero such that the inverse image of $\Delta_{x}$ under the mapping

$$
v_{*} \mapsto v^{\prime}, \text { where } v^{\prime}\left(x, v, v_{*}\right)=v-\omega_{x y}\left(\omega_{x y} \cdot\left(v-v_{*}\right)\right)
$$

has measure less than $\varepsilon_{3}$ in the set (6.8). Indeed, for every $v$ and $\omega_{x y}$ fixed, the mapping (6.12) acts as the orthogonal projection of the half-space $\left(v-v_{*}\right) \cdot \omega_{x y}<0$ onto the line $v+\mathbb{R} \omega_{x y}$. For every $x \in \Omega$ we denote by $c_{12}$ the surface measure of the solid angle of vectors $\omega_{x y}$ corresponding to $y$ in the cone (6.6). It is then easy to verify that we can find constant $c_{13}>0$ so that for, say, half of the directions $\omega_{x y}$ (measured by the surface measure on $S^{2}$ ), the intersections of the set $\Delta_{x}$ with the rays $v+\mathbb{R}_{+} \omega_{x y}$ have linear measure less than $c_{13} \varepsilon_{2}$. This implies that for certain $c_{14}>0$, and for $y$ from a set of measure at least $c_{11} / 2$, the inverse image of $\Delta_{x}$ has measure at most $c_{14} \varepsilon_{2}$ in the set (6.8).

It remains to establish the lower bound for $F\left(y, v_{*}\right)$ and the upper bound for $F\left(y, v_{*}^{\prime}\right)$ in (6.5). To obtain the former, we can use the result of Lemma 5.3 as in the discussion preceding the lemma, and for every $\varepsilon_{4}>0$ choose $c_{7}>0$ (depending on $\varepsilon_{4}$ ) so that

$$
F\left(y, v_{*}\right) \geq c_{7},
$$


for a.a. $y \in \Omega$ and for $v_{*}$ in an $y$-dependent subset of $\left\{\left|v_{*}\right| \leq R\right\}$ with complement of measure $\varepsilon_{4}$. To find an upper bound for $F\left(y, v_{*}^{\prime}\right)$ we use the integral bound obtained in next lemma. As a consequence of the integral estimate, given $(x, v) \in S^{\prime}$ and $\varepsilon_{5}>0$ we can find a set of $y$ of measure $c_{11} / 4$ such that outside of that set,

$$
F\left(y, v_{*}^{\prime}\left(x, v, y, v_{*}\right)\right) \leq c_{6},
$$

with $c_{6}$ depending on $\varepsilon_{5}$, for $v_{*}$ in a set with complement of measure $\varepsilon_{5}$ in (6.8). It is also easy to make sure that the set of $y$ can be chosen so that the first two bounds (6.5) remain valid.

Now given $\varepsilon>0$ we may take $\varepsilon_{1}+\varepsilon_{3}+\varepsilon_{4}+\varepsilon_{5}<\varepsilon$ and obtain the inequalities (6.5) for all $(x, v) \in S^{\prime}, y$ in an $x v$-dependent set of measure $c_{11} / 4$ and $v_{*}$ in an $x v y$-dependent set with complement of measure $\varepsilon$ in the set (6.8). The condition $|v| \leq R / 2$ then ensures that the volume in the $v_{*}$-space is positively bounded from below. This completes the proof.

Lemma 6.2. For every $(x, v) \in \Omega \times B(0, R / 2)$ and every $\varepsilon>0$ there is a set in $\Omega \times \mathbb{R}^{3}$ with the characteristic function $\chi_{x v}\left(y, v_{*}\right)$ such that

$$
\int_{\Omega} \int_{B_{R+}}\left(1-\chi_{x v}\right) d y d v_{*}<\varepsilon
$$

and

$$
\int_{\Omega} \int_{B_{R+}} F\left(y, v_{*}^{\prime}\left(x, v, y, v_{*}\right)\right) \chi_{x v} d y d v_{*} \leq C_{\varepsilon}
$$

where

$$
v_{*}^{\prime}\left(x, v, y, v_{*}\right)=v_{*}+\omega_{x y}\left(\omega_{x y} \cdot\left(v-v_{*}\right)\right),
$$

$\omega_{x y}$ is as in (2.5) and $B_{R+}=B_{R+}(v)$ is defined in (6.8).

Proof. We start by a remark that the statement of the lemma is not a consequence of the uniform energy bound. Indeed, for every $x, v$ and $y$ fixed, the mapping $v_{*} \mapsto v_{*}^{\prime}$ acts as the orthogonal projection of the vector $v_{*}-v$ to the plane through $v$, with the normal vector $\omega_{x y}$. Thus, in effect we need to estimate an integral of $F$ over a manifold of codimension 1 in $\Omega \times \mathbb{R}^{3}$; to accomplish this we shall use the bounds of the boundary fluxes.

We will take $\chi_{x v}$ such that for every $x$ and $v$ fixed this function is independent of $|x-y|$ and of the projection of $v_{*}-v$ onto $\omega_{x y}$. The integral in the statement of the lemma can then be estimated as

$$
C_{R} \int_{\Omega} \int_{\Pi_{R}\left(v, \omega_{x y}\right)} F\left(y, v_{*}^{\prime}\right) \chi_{x v}\left(y, v_{*}^{\prime}\right) d \pi_{v_{*}^{\prime}} d y,
$$

where $\Pi_{R}\left(v, \omega_{x y}\right)=\left\{v_{*}^{\prime} \in \mathbb{R}^{3}|| v_{*}^{\prime} \mid \leq R,\left(v-v_{*}^{\prime}\right) \cdot \omega_{x y}=0\right\}$ is a part of a plane, and $d \pi_{v_{*}^{\prime}}$ is the plane measure on $\Pi_{R}\left(v, \omega_{x y}\right)$. Changing the order of integration in (6.14) we can rewrite the above integral as

$$
\int_{\left|v_{*}^{\prime}\right| \leq R} \frac{1}{\left|v-v_{*}^{\prime}\right|} \int_{\Pi\left(x, v-v_{*}^{\prime}\right)}|x-y| F\left(y, v_{*}^{\prime}\right) \chi_{x v}\left(y, v_{*}^{\prime}\right) d \pi_{y} d v_{*}^{\prime},
$$


where $\Pi\left(x, v-v_{*}^{\prime}\right)=\left\{y \in \Omega \mid\left(v-v_{*}^{\prime}\right) \cdot \omega_{x y}=0\right\}$ is a part of a plane in the physical space, and $d \pi_{y}$ is the corresponding measure. Fixing $\delta>0$ we can use the exponential bound (2.25) together with the estimates (2.26) or (2.27) and (in the latter case) a standard Chebyshev's inequality argument to choose a set of measure $\delta$ in $\left|v_{*}^{\prime}\right| \leq R$ such that

$$
F\left(y, v_{*}^{\prime}\right) \leq C_{\delta} F\left(x^{+}\left(y, v_{*}^{\prime}\right), v_{*}^{\prime}\right),
$$

for a.a. $y \in \Omega$ and for $v_{*}^{\prime}$ in the complement of that set. Choosing $\chi_{x v}$ vanishing on the above set of measure $\delta$, estimating the factor $|x-y|$ in (6.15) by $D$, where $D$ is the diameter of $\Omega$, and changing the variables of integration from $y$ to $x^{-}\left(y, v_{*}\right)$ we obtain the bound for $(6.15)$ in the form

$$
C_{\delta} D \int_{\left|v_{*}^{\prime}\right| \leq R} \frac{1}{\left|v-v_{*}^{\prime}\right|\left|v_{*}^{\prime}\right|} \int_{\partial \Omega: v_{*}^{\prime} \cdot n_{x^{-}}<0} F\left(x^{-}, v_{*}^{\prime}\right) \chi_{x v}\left(x^{-}, v_{*}^{\prime}\right)\left|v_{*}^{\prime} \cdot n_{x^{-}}\right| d \sigma_{x^{-}} d v_{*}^{\prime} .
$$

Given $\varepsilon>0$ we take $\chi_{x v}$ vanishing for $\left|v-v_{*}^{\prime}\right| \leq \delta$ and $\left|v_{*}^{\prime}\right| \leq \delta$, choose $\delta$ small enough and use the bound for the total mass outflow to complete the proof of the lemma.

We next establish uniform bounds for the total mass of solutions to (3.12).

Lemma 6.3. Let $(F)=\left(F^{\alpha r m}\right)$ be the family of solutions to (3.12), satisfying the normalizing condition (3.13) and the bound for the entropy production (6.3). Then

$$
\iint_{\Omega \times \mathbb{R}^{3}} F(x, v) d x d v \leq C_{3},
$$

with the constant $C_{3}$ independent of $\alpha, r$ and $m$.

Proof. By the uniform energy estimate,

$$
\iint_{|v| \geq 1} F(x, v) d x d v \leq \iint_{|v| \geq 1} F(x, v)|v|^{2} d x d v \leq C_{1},
$$

where $C_{1}$ is the constant in (6.1). We shall argue by contradiction, assuming that there is a sequence $\left(F^{n}\right)=\left(F^{\alpha_{n}, r_{n}, m_{n}}\right)$, such that

$$
\mu_{n}=\iint_{|v| \leq 1} F^{n} d x d v \underset{n \rightarrow \infty}{\longrightarrow} \infty .
$$

Taking $n$ large enough and using (6.1), we see that

(6.16) $2 \iint_{|v| \leq 1} F^{n} d x d v \geq \iint_{|v| \leq 1} F^{n} d x d v+2 C_{1} \geq \iint_{\Omega \times \mathbb{R}^{3}} F^{n}\left(1+|v|^{2}\right) d x d v$. 
Applying Lemma 6.1 with $S=\Omega \times\{|v| \leq 1\}$, and using estimate (6.3) we obtain, denoting $b_{0}=|B(0,1)|$,

$$
\begin{aligned}
& C \iint_{\Omega \times \mathbb{R}^{3}} F^{n}\left(1+|v|^{2}\right) d x d v \geq \iint_{\Omega \times \mathbb{R}^{3}} e\left(F^{n}, F^{n}\right) d x d v \\
\geq & \iint_{S^{\prime}} \iint_{P_{x v}} F^{n} F_{*}^{n}\left(\frac{F^{n \prime} F_{*}^{n^{\prime}}}{F^{n} F_{*}^{n}}-\log \frac{F^{n^{\prime}} F_{*}^{n^{\prime}}}{F^{n} F_{*}^{n}}-1\right) B d y d v_{*} d x d v \\
\geq & c_{7} c_{9} c_{10}\left(\frac{c_{6} c_{8} b_{0}}{c_{7} \mu_{n}}-\log \frac{c_{6} c_{8} b_{0}}{c_{7} \mu_{n}}-1\right) \iint_{S^{\prime}} F^{n}(x, v) d x d v \\
\geq & c_{7} c_{9} c_{10}\left(\log \mu_{n}-c_{15}\right) \iint_{|v| \leq 1} F^{n}(x, v) d x d v,
\end{aligned}
$$

where $c_{15}>0$ is a constant, and $\mu_{n}$ is sufficiently large, so that $c_{6} c_{8} b_{0} / c_{7} \mu_{n}<1$. Combining (6.17) with (6.16) and letting $n$ tend to infinity we arrive at a contradiction, since we assumed $\mu_{n} \rightarrow \infty$.

In next lemma we give an equi-integrability condition for sets with uniformly small $v$-sections.

LEMMA 6.4. Let $(F)=\left(F^{\alpha r m}\right)$ be a family of solutions to (3.12), as in the previous lemma. For every $\varepsilon>0$ and every $R>0$, there exists $\delta>0$, such that for all $S \subseteq \Omega \times\{|v| \leq R\}$ and for every $F$ from the family of solutions,

$$
\iint_{S} F(x, v) d x d v<\varepsilon
$$

as soon as the measures of the $v$-sections $\left|S_{x}\right|$ are less than $\delta$ for all $x \in \Omega$.

Proof. To prove the lemma by contradiction, assume that there exists $\varepsilon>0$ such that the equi-integrability condition is violated for a sequence of solutions $\left(F^{n}\right)=\left(F^{\alpha_{n} r_{n} m_{n}}\right)$ and a sequence of sets $S_{n}$ with $\sup _{x}\left|S_{n_{x}}\right| \rightarrow 0$. By Lemma 6.1 we can pick a sequence $S_{n}^{\prime} \subseteq S_{n}$ and a sequence of sets $P_{n x y}$ with measures $\geq c_{10}$, such that for all $(x, v) \in S_{n}^{\prime}$, and all $\left(y, v_{*}\right) \in P_{n x y}$

$$
F^{n} F_{*}^{n} \geq c_{7} F^{n}
$$

and

$$
\frac{F^{n \prime} F_{*}^{n \prime}}{F^{n} F_{*}^{n}}-\log \frac{F^{n \prime} F_{*}^{n \prime}}{F^{n} F_{*}^{n}}-1 \geq \frac{c_{6} c_{8}\left|S_{n x}\right|}{c_{7} \varepsilon}-\log \frac{c_{6} c_{8}\left|S_{n x}\right|}{c_{7} \varepsilon}-1 \geq \log \frac{1}{\left|S_{n x}\right|}-c_{15},
$$

for $n$ large enough, such that $c_{6} c_{8}\left|S_{n x}\right| / c_{7} \varepsilon<1$. Using the estimate for the 
total mass of $F^{n}$ and then arguing as in the previous lemma, we obtain

$$
\begin{aligned}
& \frac{C_{2}\left(C_{3}+C_{1}\right)}{\varepsilon} \iint_{S_{n}} F^{n}(x, v) d x d v \\
\geq & C_{1} \iint_{\Omega \times \mathbb{R}^{3}} F^{n}(x, v)\left(1+|v|^{2}\right) d x d v \\
\geq & \iint_{\Omega \times \mathbb{R}^{3}} e\left(F^{n}, F^{n}\right) d x d v \\
\geq & \iint_{S_{n}^{\prime}} \iint_{P_{n x y}} F^{n} F_{*}^{n}\left(\frac{F^{n^{\prime}} F_{*}^{n^{\prime}}}{F^{n} F_{*}^{n}}-\log \frac{F^{n \prime} F_{*}^{n \prime}}{F^{n} F_{*}^{n}}-1\right) B d y d v_{*} d x d v \\
\geq & c_{7} c_{9} c_{10}\left(\log \frac{1}{\sup _{x}\left|S_{n x}\right|}-c_{15}\right) \iint_{S_{n}} F^{n}(x, v) d x d v .
\end{aligned}
$$

We arrive at a contradiction, since by assumption, $\sup _{x}\left|S_{n x}\right| \rightarrow 0$.

We have now established uniform bounds from above and from below for the weighted mass $\mu(F)$, which allow us to pass to the original formulation of the problem (3.3), (3.4), with $\alpha>0$ and the collision term $Q_{r m}(f, f)$ retaining the $r$ and $m$ truncations as in (4.1). Solutions $f=f^{\alpha r m}=F^{\alpha r m} / \mu\left(F^{\alpha r m}\right)$ now have unit weighted mass (condition (3.11) with $\kappa=1$ ), and the boundary traces satisfying the inequalities

$$
0<c_{16} \leq \iint_{\partial \Omega^{ \pm}} f_{ \pm}\left|v \cdot n_{x}\right| d \sigma_{x} d v \leq c_{17},
$$

for certain $c_{16}, c_{17}>0$, uniformly in $\alpha, r$ and $m$. The lower bound for the total mass provided by Lemma 5.3 also implies that the equi-integrability condition of Lemma 6.4 can be transfered to solutions $f=f^{\alpha r m}$.

We next show that the family $(f)=\left(f^{\alpha r m}\right)$ is relatively weakly compact in $L^{1}\left(\Omega \times \mathbb{R}^{3}\right)$, for which it remains to show the following equi-integrability condition. For every $R>0$ and every $\varepsilon>0$ there exists $\delta>0$ such that for all $f$ from the family of solutions,

$$
\int_{\Delta} \int_{|v| \leq R} f(x, v) d v d x<\varepsilon,
$$

as soon as the set $\Delta \subseteq \Omega$ has measure less than $\delta$. Indeed, having established (6.19) we can verify the conditions for the equi-integrability over $\Omega \times \mathbb{R}^{3}$, as follows. Using the energy bound of Lemma 3.1 the integrals of $f$ over $\Omega \times\{|v| \geq R\}$ are uniformly bounded by $C_{1} / R^{2}$. Also, given $R>0$, and a measurable set $S$ in $\Omega \times\{|v| \leq R\}$, we can split its $x$-projection into two parts as follows. In the first part we choose those $x$ for which the $v$-sections have volume less than $|S|^{1 / 2}$, and the remainder is such that its $x$-projection has volume less than $|S|^{1 / 2}$ in $\Omega$. By Lemma 6.4, the integral of $f$ over the first set can be made as small as desired, if $|S|$ is small enough. To estimate the second part of the integral, the equi-integrability condition (6.19) is sufficient.

We shall first use Lemma 6.4 to establish an equi-integrability condition for the mass outflow. 
LeMma 6.5. Let $(f)=\left(f^{\alpha r m}\right)$ be the family of solutions to (3.3), (3.4), satisfying (3.11) and (6.18). Then for every $R>0$ and every $\varepsilon>0$ there exists $\delta>0$, such that for all $f$ from the family of solutions,

$$
\int_{\Delta} \int_{\left\{v \cdot n_{x}<0,|v| \leq R\right\}} f_{-}(x, v)\left|v \cdot n_{x}\right| d v d \sigma_{x}<\varepsilon,
$$

provided the area of $\Delta \subseteq \partial \Omega$ is less than $\delta$.

Proof. We give a proof by contradiction. Assuming that the conclusion of the lemma is false, we can pick an $\varepsilon>0$, a sequence of solutions $\left(f^{n}\right)$ and a sequence of sets $\left(\Delta_{n}\right)$ in $\partial \Omega$, such that $\left|\Delta_{n}\right| \rightarrow 0$, and for all $n$, inequality (6.20) is violated for $f=f^{n}$ and $\Delta=\Delta_{n}$. Along those characteristics for which $x^{+} \in \Delta_{n}$ and $\int_{-s^{-}}^{s^{-}} \nu^{\sharp} d s \leq a$, with $a$ constant, we can estimate solutions inside the domain by the incoming traces using (2.25):

$$
f(x, v) \geq e^{-a} f_{+}\left(x^{+}, v\right) .
$$

Using the uniform bounds of the total mass and energy and estimates (2.26) and (2.27), the above inequality is valid for every $R>0$ and $\eta>0$, with $a$ dependent on $R$ and $\eta$, for a.a. $x^{+} \in \partial \Omega$ and for $v$ in a set with complement of measure $\eta$ in $\left\{|v| \leq R, v \cdot n_{x^{+}}>0\right\}$. Further, picking $\theta \in(0,1)$ and $0<\sigma<d_{\theta} / 2$, so that (2.12) is satisfied, we see that the solid angle in the velocity space, in which $\Delta_{n}$ is 'visible' from a point $x \in \Omega$ is less than $c\left|\Delta_{n}\right| / \sigma^{2}$, for all $\left|x-x^{+}\right|>\sigma$. (The last condition defines a set of positive measure in $\Omega$ for $\sigma$ small enough, because of the cone condition.) Thus, by Lemma 6.4,

$$
\int_{\Omega} \int_{\left\{|v| \leq R, x^{+} \in \Delta_{n},\left|x-x^{+}\right|>\sigma\right\}} f(x, v) d v d x \underset{n \rightarrow \infty}{\longrightarrow} 0 .
$$

In the above integral, we split the $x$-integration into the integration over $\partial \Omega$ and along the characteristic in the direction of $v$, and use (6.21), to estimate it from below as follows:

$$
e^{-a} \int_{\Delta_{n}} d \sigma_{x}+\int_{\left\{|v| \leq R, \int v^{\sharp} d s \leq a\right\}} d v\left|v \cdot n_{x^{+}}\right| f_{+}\left(x^{+}, v\right) \int_{-s^{+}+\sigma /|v|}^{s^{-}} d s .
$$

Restricting the directions of $v$ to those in the cone $C_{\theta, d_{\theta}}\left(x^{+}\right)$we have the inequalities

$$
\left|v \cdot n_{x^{+}}\right| \geq \theta|v| \text { and } \quad \int_{-s^{+}+\sigma /|v|}^{s^{-}} d s \geq \frac{\sigma}{|v|} .
$$

Using the boundary condition (3.4) and the lower bound (2.19) we can further estimate (6.23) from below as

$$
\sigma \theta e^{-a} \int_{\Delta_{n}} d \sigma_{x} \int_{w \cdot n_{x}<0} d w\left|w \cdot n_{x}\right| f_{-}(x, w) \int_{\left\{|v| \leq R, v \cdot n_{x}>\theta|v|, \int v^{\sharp} d s \leq a\right\}} \Phi_{1}(|v|) d v .
$$


For every $\theta$ fixed, the volume of integration over $v$ in the integral $d v$ is bounded away from zero if we choose $\eta$ small enough. Since $\Phi_{1}(|v|)$ is a Maxwellian, the integral over $v$ is positively bounded from below, independently of $n$. Therefore, we obtain a contradiction to (6.22) since we assumed that (6.20) is false for all $n$.

REMARK. The proof of the lemma uses only the inequality $f_{+} \geq \mathcal{R} f_{-}$on the boundary and estimate of the type (2.25), and so, the result holds in the generality of Section 5, provided the result of Lemma 6.4 could be established for solutions of (5.1).

Next lemma provides an argument which establishes the equi-integrability condition (6.19) and completes the proof of weak compactness. The argument, based on the bound (4.18) and the regularizing properties of the inverse transport operator applies in the cases of both 'hard' and 'soft' interactions. In the 'hard' interactions case it offers a shorter proof of Lemma 6.8 in [7].

LEMMA 6.6. Let $\left(f^{\alpha r m}\right)$ be the family of solutions of (3.3), (3.4), as in the previous lemma. Then for each $\varepsilon>0$ there is a $\delta>0$, such that for all $f \in\left(f^{\alpha r m}\right)$ condition (6.19) holds, provided the measure of the set $\Delta \subseteq \Omega$ is less than $\delta$.

Proof. Given $\varepsilon>0$, by the equi-integrability condition of Lemma 6.4, we can take a constant $r_{1}>0$ so small that for all $f$ from the family of solutions,

$$
\int_{\Omega} \int_{|v| \leq r_{1}} f(x, v) d v d x<\varepsilon / 8 .
$$

Thus, setting $\chi_{r_{1}}=\chi_{\left\{|v|>r_{1}\right\}}$, it suffices to prove an inequality of type (6.19) for $\chi_{r_{1}} f$.

Using (2.24) we represent $f(x, v)$ in the exponential form as

$$
f(x, v)=f_{+}\left(x^{+}, v\right) e^{-\int_{-s^{+}}^{0} v^{\sharp} d s}+\int_{-s^{+}}^{0} Q^{+}(f, f)^{\sharp} e^{-\int_{s}^{0} v^{\sharp} d \tau} d s .
$$

Integrating (6.24) over $\Delta \times\{|v| \leq R\}$, we shall estimate separately the terms corresponding to the boundary data and collisions in the volume. In the boundary term, we estimate the exponential factor as 1 ; the remaining integral can be written as follows:

$$
\begin{aligned}
& \iint_{\partial \Omega^{+}} \chi_{r_{1}}(v) f_{+}\left(x^{+}, v\right) \int_{0}^{s^{-}\left(x^{+}, v\right)} \chi_{\Delta}\left(x^{+}+s v\right) d s\left|v \cdot n_{x^{+}}\right| d \sigma_{x}+d v \leq C \alpha|\Delta| \\
+ & \iint_{\partial \Omega^{-}} f_{-}(x, w) \int_{v \cdot n_{x}>0} k(x, v, w) \chi_{r_{1}}(v) \int_{0}^{s^{-}(x, v)} \chi_{\Delta}^{\sharp} d s d v\left|w \cdot n_{x}\right| d \sigma_{x} d w,
\end{aligned}
$$

where $\chi_{\Delta}^{\sharp}=\chi_{\Delta}(x+s v)$. Here we used the boundary condition (3.4) and estimated the source term $S(x, v)$ pointwise by its maximum. Further, for 
every $x \in \partial \Omega$ and $v \in\{|v| \leq R\}$ fixed we split $\Delta$ into two sets $\Delta_{1}$ and $\Delta_{2}$, so that

$$
\int_{0}^{s^{-}(x, v)} \chi_{\Delta_{1}}^{\sharp} d s \leq \frac{|\Delta|^{1 / 2}}{|v|} \leq \frac{|\Delta|^{1 / 2}}{r_{1}},
$$

and the area of the projection of $\Delta_{2}$ onto the plane orthogonal to $v$ is less than $|\Delta|^{1 / 2}$. Because of the normalization (2.16) and the uniform bound (6.18), the integral with $\chi_{\Delta_{1}}$ can be made less than $\varepsilon / 8$ by taking $|\Delta|$ small enough. On the other hand,

$$
\iint_{\Delta_{2} \times\{|v| \leq R\}} \chi_{r_{1}} f_{+}\left(x^{+}, v\right) d x d v \leq \frac{D}{r_{1}} \iint_{\partial \Omega^{+}} \chi_{r_{1}} f_{+}(x, v) \chi_{\Delta_{2}^{+}}\left|v \cdot n_{x}\right| d \sigma_{x} d v
$$

where $\Delta_{2}^{+}$is a set of characteristics satisfying

$$
\iint_{\partial \Omega^{+}} \chi_{\Delta_{2}^{+}}\left|v \cdot n_{x}\right| d \sigma_{x} d v \leq c_{R}|\Delta|^{1 / 2}
$$

The set $\Delta_{2}^{+}$can then be split into two parts, one with measure $|\Delta|^{1 / 4}$ on $\partial \Omega$ and the rest such that for every $x$ the measure in the velocity space is $c_{R}|\Delta|^{1 / 4}$. Estimating the integrals of the two parts analogously to (6.25) and using Lemma 6.5 for the first part and condition (2.16) for the second, we can ensure that the integral with $\chi_{\Delta_{2}}$ is less than $\varepsilon / 8$.

Further, we estimate the second term on the right-hand side of (2.24), for which we first notice that in view of the bounds (2.26) and (2.27) and the uniform bounds for the total mass and energy,

$$
\int_{|v| \leq R}\left\{\sup _{x \in \Omega} v(x, v)\right\} d v \leq C_{R}
$$

for both 'hard' and 'soft' interactions. Thus, choosing $a>0$ sufficiently large, we can pick a set of $v \in\{|v| \leq R\}$, with characteristic function $\chi_{a}(v)$, such that $\chi_{a} v(f) \leq a$, and the complement of that set is as small as desired. Lemma 6.4 then implies that

$$
\iint_{\Omega \times \mathbb{R}^{3}} f\left(1-\chi_{a}\right) d x d v<\varepsilon / 8
$$

for $a$ sufficiently large. Since the exponential factors in (6.24) are bounded by 1 , it suffices to prove a condition of type (6.19) for

$$
\chi_{r_{1}} \chi_{a} \int_{-s^{+}}^{0} Q_{r m}^{+}(f, f)^{\sharp} d s
$$

for every $r_{1}>0$ and $a>0$. Using the bound (4.18) with $K$ large enough we can replace the 'gain' term in (6.27) by the 'loss' term $f v(f)$. Indeed, integrating (6.27) over $\Delta \times\{|v| \leq R\}$ and using that for $r_{1}>0$ the integration along characteristic in (6.27) is a bounded operator from $L^{1}\left(\Omega \times \mathbb{R}^{3}\right)$ to itself, one 
can make the contribution of the entropy production term in (4.18) as small as desired by choosing $K$ large enough. Further, since $\chi_{a} v(f)$ is bounded uniformly by $a$, the problem is reduced to estimating the integral (6.27) with $f$ instead of $Q_{r m}^{+}(f, f)$. This can be accomplished by arguing as follows.

We write $x=p+z v /|v|$, where $p$ is the projection of $x$ onto the plane $v^{\perp}=\left\{p \in \mathbb{R}^{3} \mid(p \cdot v)=0\right\}$, and accordingly,

$$
\begin{aligned}
& \iint_{\Delta \times\{|v| \leq R\}} f d x d v \\
= & \int_{|v| \leq R} d v \int_{v^{\perp}} d p \int_{-\infty}^{\infty} d z \chi_{\Delta}\left(p+z \frac{v}{|v|}\right) \int_{-s^{+}}^{0} d s \chi_{r_{1}} f\left(p+z \frac{v}{|v|}+s v, v\right) .
\end{aligned}
$$

For those characteristics for which the lengths of the sections of $\Delta$ (given by the integrals $\left.\int_{-\infty}^{\infty} \chi_{\Delta}(p+z v /|v|) d z\right)$ are less than $|\Delta|^{1 / 4}$, we obtain:

$$
\begin{aligned}
& \int_{-\infty}^{\infty} d z \chi_{\Delta}\left(p+z \frac{v}{|v|}\right) \int_{-s^{+}}^{0} d s \chi_{r_{1}} f\left(p+z \frac{v}{|v|}+s v, v\right) \\
\leq & \int_{-\infty}^{\infty} d z \chi_{\Delta}\left(p+z \frac{v}{|v|}\right) \frac{1}{|v|} \int_{-\infty}^{\infty} d \zeta \chi_{\Omega}\left(p+\zeta \frac{v}{|v|}\right) \chi_{r_{1}} f\left(p+\zeta \frac{v}{|v|}, v\right) \\
\leq & \frac{1}{r_{1}}|\Delta|^{1 / 4} \int_{-\infty}^{\infty} d \zeta \chi_{\Omega}\left(p+\zeta \frac{v}{|v|}\right) \chi_{r_{1}} f\left(p+\zeta \frac{v}{|v|}, v\right) .
\end{aligned}
$$

Denoting by $\chi^{(1)}$ the characteristic function of the above set of $(v, p)$, we see that the integral of the type (6.28) with $\chi^{(1)} f$ can be bounded as follows, using (6.29) and the uniform mass bound,

$$
\begin{aligned}
& \frac{1}{\delta}|\Delta|^{1 / 4} \int_{|v| \leq R} d v \int_{v^{\perp}} d p \int_{-\infty}^{\infty} d \zeta \chi_{\Omega}\left(p+\zeta \frac{v}{|v|}\right) f\left(p+\zeta \frac{v}{|v|}, v\right) \\
= & \frac{1}{\delta}|\Delta|^{1 / 4} \iint_{\Omega \times \mathbb{R}^{3}} f(x, v) d x d v \leq C|\Delta|^{1 / 4}<\frac{\varepsilon}{8 a},
\end{aligned}
$$

if $|\Delta|$ is small enough. For the remaining part of $(v, p)$, with the characteristic function $\chi^{(2)}$, we obtain, instead of (6.29),

$$
\begin{aligned}
& \chi^{(2)} \int_{-\infty}^{\infty} d z \chi_{\Delta}\left(p+z \frac{v}{|v|}\right) \int_{-s^{+}}^{0} d s \chi_{\delta} f\left(p+z \frac{v}{|v|}+s v, v\right) \\
\leq & \frac{D}{\delta} \chi^{(2)} \int_{-\infty}^{\infty} d \zeta \chi_{\Omega}\left(p+\zeta \frac{v}{|v|}\right) \chi_{\delta} f\left(p+\zeta \frac{v}{|v|}, v\right) .
\end{aligned}
$$

Since the sections of $\Delta$ for all characteristics from the second set have lengths at least $|\Delta|^{1 / 4}$, we can estimate from above the solid angle $\Sigma\left(x^{\sharp}\right)$ of directions for such characteristics passing through a given point $x^{\sharp}$ in $\Omega$. Indeed, writing the 
volume of $\Delta$ as an integral of $\chi_{\Delta}$ and transforming to the spherical coordinates with center at $x^{\sharp}$, we obtain

$$
|\Delta| \geq \int_{\Sigma\left(x^{\sharp}\right)} d \omega \int_{0}^{\infty} \rho^{2} d \rho \chi_{\Delta}\left(x^{\sharp}+\rho \omega\right) \geq\left|\Sigma\left(x^{\sharp}\right)\right| \int_{0}^{\frac{1}{2}|\Delta|^{1 / 4}} \rho^{2} d \rho=\frac{1}{24}\left|\Sigma\left(x^{\sharp}\right)\right||\Delta|^{3 / 4},
$$

where we used that the integral in the spherical coordinates takes its least value when all sections are the segments of length $|\Delta|^{1 / 4}$ centered at $x^{\sharp}$. The last inequality implies that $\left|\Sigma\left(x^{\sharp}\right)\right|$ is uniformly in $x^{\sharp} \in \Omega$ bounded as follows:

$$
\left|\Sigma\left(x^{\sharp}\right)\right| \leq 24|\Delta|^{1 / 4} .
$$

Thus, integrating (6.30) over $(v, p)$, we obtain

$$
\begin{aligned}
& \int_{\Delta} \int_{|v| \leq R} \chi^{(2)} \int_{-s^{+}}^{0} \chi_{r_{1}} f^{\sharp} d s d x d v \\
\leq & \frac{D}{r_{1}} \int_{|v| \leq R} d v \int_{v^{\perp}} d p \chi^{(2)} \int_{-\infty}^{\infty} d \zeta \chi_{\Omega}\left(p+\zeta \frac{v}{|v|}\right) f\left(p+\zeta \frac{v}{|v|}, v\right) \\
= & \frac{D}{r_{1}} \int d x^{\sharp} \int_{\left\{\frac{v}{|v|} \in \Sigma\left(x^{\sharp}\right)\right\}} d v f\left(x^{\sharp}, v\right) .
\end{aligned}
$$

Lemma 6.4 now implies, in view of the condition (6.31) that the last integral can be made less than $\varepsilon / 8 a$, uniformly in $f$, by choosing $|\Delta|$ small. This completes the proof of the lemma.

\section{7. - Proofs of Theorems $2.1-2.3$}

PROOF OF THEOREM 2.1. Using the weak compactness of the approximations, established in the previous section, we can pick a subsequence $f^{n}=f^{r_{n}, m_{n}, \alpha_{n}}$ with $r_{n} \rightarrow 0, m_{n} \rightarrow \infty$ and $\alpha_{n} \rightarrow 0$, so that $f^{n}$ converges weakly in $L^{1}$ to a limit function $f$. Further, inequality (2.26) for $0 \leq \beta<2$ and the uniform bound for the energy of $f$ imply that the sequence of the 'loss' terms $f^{n} v_{n}\left(f^{n}\right)$ is weakly compact in $L^{1}$. In view of the entropy production estimate of Lemma 3.2 and inequality (4.18), the weak compactness of the 'gain' terms also follows. We now can, following the arguments of [7], for every $\psi \in L^{\infty}\left(\Omega \times \mathbb{R}^{3}\right)$ with $v \cdot \nabla_{x} \psi \in L^{\infty}\left(\Omega \times \mathbb{R}^{3}\right)$, pass to the limit $n \rightarrow \infty$ in the weak form

$$
\begin{aligned}
& \iint_{\partial \Omega^{-}} f_{-}^{n} \psi_{-}\left|v \cdot n_{x}\right| d \sigma_{x} d v-\iint_{\partial \Omega^{+}} f_{+}^{n} \psi_{+}\left|v \cdot n_{x}\right| d \sigma_{x} d v \\
= & \iint_{\Omega \times \mathbb{R}^{3}} f^{n} v \cdot \nabla_{x} \psi d x d v+\iint_{\Omega \times \mathbb{R}^{3}}\left(Q_{n}\left(f^{n}, f^{n}\right)-\alpha_{n} f^{n}\right) \psi d x d v,
\end{aligned}
$$

where $Q_{n}$ is the collision operator (2.2) with the kernel (4.1) corresponding to the sequence $r_{n}, m_{n}$. This allows us to conclude in the case of 'hard' interactions. 
The proof in the case of 'soft' interactions, $-3<\beta<0$, requires significantly more effort, which is mainly due to the fact that we do not have a priori bounds for $Q_{n}\left(f^{n}, f^{n}\right)$ in $L^{1}$ (in fact, in the end we are only able to prove that the limit solutions satisfy the iterated integral form (2.30)). We recall that we identify the characteristics with the points in the set $\partial \Omega^{-}$, equipped with the measure $\left|v \cdot n_{x}\right| d \sigma_{x} d v$, so that identity (2.29) holds. For every $R>0$ we define

$$
\partial \Omega_{R}^{-}=\left\{(x, v) \in \partial \Omega^{-}|| v \mid \leq R\right\},
$$

and for every $S \subseteq \partial \Omega^{-}$we set

$$
S^{\sharp}=\left\{(x, v) \in \Omega \times \mathbb{R}^{3} \mid\left(x^{-}(x, v), v\right) \in S\right\} .
$$

We then establish the following compactness result.

LEMMA 7.1. Let $f^{n}$ be a sequence of solutions to (2.1), converging weakly in $L^{1}\left(\Omega \times \mathbb{R}^{3}\right)$, and let $v\left(f^{n}\right)$ and $Q^{+}\left(f^{n}, f^{n}\right)$ be defined by (2.2) and (2.3). Assume also that $f^{n}$ have boundary traces $f_{-}^{n}$ which satisfy

$$
\iint_{\partial \Omega^{-}} f_{-}^{n}\left|v \cdot n_{x}\right| d \sigma_{x} d v \leq C,
$$

uniformly in $n$. For every $R>0$ and every $\varepsilon>0$ there is a set of characteristics $E_{\varepsilon, R} \subseteq \partial \Omega_{R}^{-}$of measure at most $\varepsilon$, such that the sequence $\chi_{\varepsilon} f^{n} v\left(f^{n}\right)$, where $\chi_{\varepsilon}=$ $\chi_{\varepsilon, R}$ is the characteristic function of the set $\left(\partial \Omega_{R}^{-} \backslash E_{\varepsilon, R}\right)^{\sharp}$, is weakly precompact in $L^{1}\left(\Omega \times \mathbb{R}^{3}\right)$.

Proof. To construct the sets $E_{\varepsilon, R}$ we first notice that the sequence $v\left(f^{n}\right)$ converges strongly in $L_{\mathrm{loc}}^{1}\left(\Omega \times \mathbb{R}^{3}\right)$, and therefore, the sequence of the integrals $\int_{-s^{+}(x, v)}^{0} v\left(f^{n}\right)^{\sharp} d s$ converges strongly in $L_{\text {loc }}^{1}\left(\partial \Omega^{-},\left|v \cdot n_{x}\right| d \sigma_{x} d v\right)$. Thus, fixing $R>0$ and $\varepsilon>0$, we can take a set $E_{\varepsilon, R}^{(1)} \subseteq \partial \Omega_{R}^{-}$of measure $\varepsilon$, such that the the integrals along characteristics converge uniformly as functions of $(x, v)$ in the complement of $E_{\varepsilon, R}^{(1)}$ and are uniformly bounded by a constant $a_{\varepsilon}$. Next, considering $|v| \leq R$ and using the cone condition for $\Omega$, we can take $\tau_{\varepsilon}>0$ and a set of characteristics $E_{\varepsilon, R}^{(2)} \subseteq \partial \Omega^{-}$of measure $\varepsilon$ such that $s^{-}+s^{+}>\tau_{\varepsilon}$ for all characteristics in its complement. Further, we notice that the strong convergence of $v\left(f^{n}\right)$ implies that

$$
\int_{-\eta s^{+}(x, v)}^{0} v\left(f^{n}\right)^{\sharp} d s \underset{\eta \rightarrow 0}{\longrightarrow} 0,
$$

in $L_{\mathrm{loc}}^{1}\left(\partial \Omega^{-},\left|v \cdot n_{x}\right| d \sigma_{x} d v\right)$, uniformly in $n$ and hence, uniformly in $(x, v) \in$ $\partial \Omega_{R}^{-}$outside a set $E_{\varepsilon, R}^{(3)}$ of measure $\varepsilon$. Finally, we take $\delta>0$ so small that the set $E_{\varepsilon}^{(4)}=\left\{(x, v) \in \partial \Omega^{-}|| v \mid<\delta\right\}$ has measure $\varepsilon$. We then set $E_{\varepsilon, R}=$ $E_{\varepsilon, R}^{(1)} \cap E_{\varepsilon, R}^{(2)} \cap E_{\varepsilon, R}^{(3)} \cap E_{\varepsilon}^{(4)}$. 
We next show that $\chi_{\varepsilon} f^{n} v\left(f^{n}\right)$ is weakly compact in $L^{1}\left(\Omega \times \mathbb{R}^{3}\right)$ by using the equi-integrability criterion. First of all, the integrals over the part of $\Omega \times \mathbb{R}^{3}$ close to $\partial \Omega^{-}$can be made uniformly small. More precisely, integrating along a characteristic from $-\eta s^{+}(x, v)$ to 0 and then over $\partial \Omega^{-}$, we get using (2.25),

$$
\begin{aligned}
& \iint_{\partial \Omega^{-}}\left|v \cdot n_{x}\right| d \sigma_{x} d v \int_{-\eta s^{+}(x, v)}^{0}\left(\chi_{\varepsilon} f^{n} v\left(f^{n}\right)\right)^{\sharp} d s \\
\leq & e^{a_{\varepsilon}} \iint_{\partial \Omega^{-}} d v d \sigma_{x}\left|v \cdot n_{x}\right| \chi_{\varepsilon} f_{-}^{n}(x, v) \int_{-\eta s^{+}(x, v)}^{0} v\left(f^{n}\right)^{\sharp} d s .
\end{aligned}
$$

Since the total mass outflow is bounded uniformly in $n$, the uniform convergence (7.2) implies that the right-hand side of (7.3) tends to zero as $\eta \rightarrow 0$, uniformly in $n$. Fixing $\eta>0$ let us now take a measurable set $\Delta$ in the remainder of $\Omega \times\{|v| \leq R\}$. Then the set of characteristics passing through $\Delta$ can be split into two parts: $S_{1} \subseteq \partial \Omega^{-}$which has measure $|\Delta|^{1 / 2}$, and $S_{2} \subseteq \partial \Omega^{-}$, in which for all $(x, v)$,

$$
\int_{-s^{+}(x, v)}^{0} \chi_{\Delta}^{\sharp} d s<|\Delta|^{1 / 2} .
$$

For the first set, we obtain, using (2.25) and estimating the infimum by the mean value,

$$
\begin{aligned}
& \chi_{\varepsilon} f^{n \sharp}(s) v_{n}\left(f^{n}\right)^{\sharp}(s) \leq e^{a_{\varepsilon}} \chi_{\varepsilon} v_{n}\left(f^{n}\right)^{\sharp}(s) \inf _{-\eta s^{+}<t<s^{-}} f^{n \sharp}(t) \\
\leq & e^{a_{\varepsilon}} \chi_{\varepsilon} v_{n}\left(f^{n}\right)^{\sharp}(s) \frac{1}{\eta s^{+}(x, v)} \int_{-\eta s^{+}(x, v)}^{0} f^{n \sharp}(t) d t,
\end{aligned}
$$

where $0<s<-\eta s^{+}(x, v)$ and $(x, v) \in S_{1}$. Integrating with respect to $s$ and then over the characteristics in $S_{1}$, we can conclude that for all $\zeta>0$

$$
\begin{aligned}
& \iint_{S_{1}}\left|v \cdot n_{x}\right| d \sigma_{x} d v \chi_{\varepsilon} \int_{-s^{+}(x, v)}^{-\eta s^{+}(x, v)}\left(f^{n} v_{n}\left(f^{n}\right)\right)^{\sharp} d s \\
\leq & \frac{a_{\varepsilon} e^{a_{\varepsilon}}}{\eta \tau_{\varepsilon}} \iint_{S_{1}}\left|v \cdot n_{x}\right| d \sigma_{x} d v \chi_{\varepsilon} \int_{-\eta s^{+}(x, v)}^{0} f^{n \sharp}(t) d t<\zeta,
\end{aligned}
$$

provided $|\Delta|$ is small enough, based on the equi-integrability of $f^{n}$.

For the characteristics from the set $S_{2}$, we use the estimate of the type (7.3) to find

$$
\begin{aligned}
& \iint_{S_{2}}\left|v \cdot n_{x}\right| d \sigma_{x} d v \int_{-s^{+}(x, v)}^{0}\left(\chi_{\varepsilon} \chi_{\Delta} f^{n} v\left(f^{n}\right)\right)^{\sharp} d s \\
\leq & e^{a_{\varepsilon}} \iint_{S_{2}}\left|v \cdot n_{x}\right| d \sigma_{x} d v \chi_{\varepsilon} f_{-}^{n}\left(x^{-}, v\right) \int_{-s^{+}(x, v)}^{0}\left(\chi_{\Delta} v\left(f^{n}\right)\right)^{\sharp} d s
\end{aligned}
$$


and notice that the integrals of $\left(\chi_{\Delta} v\left(f^{n}\right)\right)^{\sharp}$ along characteristics converge to zero uniformly in $x, v$ and $n$, as $|\Delta| \rightarrow 0$. Indeed, we can write

$$
\begin{aligned}
& \int_{-s^{+}(x, v)}^{0}\left(\chi_{\Delta} v\left(f^{n}\right)\right)^{\sharp} d s \\
= & \iint_{\Omega \times \mathbb{R}^{3}} f^{n}\left(y, v_{*}\right)\left|v-v_{*}\right|^{\beta} \int_{-s^{+}(x, v)}^{0} \chi_{\Delta}^{\sharp} h(\cos \theta) b(x+v s, y) d s d y d v_{*},
\end{aligned}
$$

where $\theta$ is the angle between $x+v s-y$ and $v-v_{*}$. Since the product $h(\cos \theta) b(x+v s, y)$ is a bounded function and $|v|>\delta$ by the definition of $E_{\varepsilon}^{(4)}$, the inner integral along a characteristic tends to zero as $|\Delta| \rightarrow 0$, uniformly in $x, v, y$ and $v_{*}$. This proves that $\chi_{\varepsilon} f^{n} v_{n}\left(f^{n}\right)$ is weakly compact in $L^{1}\left(\Omega \times \mathbb{R}^{3}\right)$ and completes the proof of the lemma.

REMARK.

1) It is easy to see that the proof of Lemma 7.1 holds for the Povzner equations with the truncated kernels $B_{r m}$ defined in (4.1).

2) As an immediate consequence of Lemma 7.1 and the entropy production estimate we obtain that the sequence $\chi_{\varepsilon} Q^{+}\left(f^{n}, f^{n}\right)$ is weakly compact, using inequality (4.18).

We further establish a compactness result for the boundary traces, which will allow us to pass to the limit in the boundary condition (2.14). The author is thankful to A. Nouri for communicating the idea of the proof [30].

LEMMA 7.2. Let $f_{ \pm}^{n}$ be two sequences of functions uniformly bounded in $L^{1}\left(\partial \Omega^{ \pm},\left(1+|v|^{2}\right)\left|v \cdot n_{x}\right| d \sigma_{x} d v\right)$, satisfying the boundary condition (2.14) and the a priori bound

$$
\iint_{\Omega \times \mathbb{R}^{3}} \mathcal{E}_{M_{\mathrm{w}}}\left(f^{n}\right)\left|v \cdot n_{x}\right| d \sigma_{x} d v \leq C,
$$

where $\mathcal{E}_{M_{\mathrm{w}}}\left(f^{n}\right)$ is defined in (3.10). Further, assume that the sequence

$$
\rho^{n}(x)=\int_{v \cdot n_{x}<0} f^{n}(x, v)\left|v \cdot n_{x}\right| d v
$$

is weakly compact in $L^{1}(\partial \Omega)$. Then $f_{ \pm}^{n}$ are weakly compact in $L^{1}\left(\partial \Omega^{ \pm}, \mid v\right.$. $\left.n_{x} \mid d \sigma_{x} d v\right)$.

Proof. The compactness of $f_{+}^{n}$ follows immediately from the compactness of $\rho^{n}$ and the bound

$$
f_{+}^{n}(x, v) \leq \rho^{n}(x) \Phi_{2}(|v|), \quad(x, v) \in \partial \Omega^{+},
$$

which we have in view of the assumption (2.19). Therefore, it remains to establish the weak compactness of $f_{-}^{n}$. Using the nonnegativity of $\mathcal{E}_{M_{\mathrm{w}}}\left(f^{n}\right)$ we can write, for every measurable set $S \in \partial \Omega$,

$$
\int_{S} \int_{v \cdot n_{x}>0} \mathcal{E}_{M_{\mathrm{w}}}\left(f^{n}\right)\left|v \cdot n_{x}\right| d \sigma_{x} d v \leq C .
$$


Arguing as in the proof of Lemma 3.2, we obtain,

$$
\int_{S} \int_{v \cdot n_{x}<0} f_{-}^{n} \log f_{-}^{n}\left|v \cdot n_{x}\right| d \sigma_{x} d v \leq C+\int_{S} \int_{v \cdot n_{x}>0} f_{+}^{n} \log f_{+}^{n}\left|v \cdot n_{x}\right| d \sigma_{x} d v .
$$

In the last integral we use inequality (7.4) and consider separately the cases when $\rho^{n}(x)<1$ and $\rho^{n}(x)>1$. In the first case the integral is bounded by

$\iint_{\partial \Omega^{+}} f_{+}^{n} \log \Phi(|v|)\left|v \cdot n_{x}\right| d \sigma_{x} d v \leq C \iint_{\partial \Omega^{+}} f_{+}^{n}\left(1+|v|^{2}\right)\left|v \cdot n_{x}\right| d \sigma_{x} d v \leq C_{1}$.

In the other case, we can use the bound

$$
C \int_{S} \rho^{n} \log \rho^{n} d \sigma_{x}
$$

We now establish that $f_{-}^{n}$ is weakly compact by using the equi-integrability criterion. Taking $\Delta \subseteq \partial \Omega^{-}$with finite measure, we have for every $K>1$ and $c>1$,

$$
\begin{aligned}
& \int_{\Delta} \int_{v \cdot n_{x}<0} f_{-}^{n}\left|v \cdot n_{x}\right| d \sigma_{x} d v \leq|\Delta| K \\
& +\iint_{\left\{(x, v) \in \Delta, f_{-}^{n}>K\right\}} f_{-}^{n}\left|v \cdot n_{x}\right| d \sigma_{x} d v \\
& \leq|\Delta| K+\frac{1}{\log K} \iint_{\left\{(x, v) \in \Delta, f_{-}^{n}>K, \rho(x) \leq c\right\}} f_{-}^{n} \log f_{-}^{n}\left|v \cdot n_{x}\right| d \sigma_{x} d v \\
& \quad+\iint_{\left\{(x, v) \in \Delta, \rho^{n}(x) \leq c\right\}} f_{-}^{n} \log f_{-}^{n}\left|v \cdot n_{x}\right| d \sigma_{x} d v \\
& \leq|\Delta| K+\frac{1}{\log K}(C+|\partial \Omega| c \log c)+\int_{\left\{\rho^{n}(x)>c\right\}} \rho^{n}(x) d \sigma_{x} .
\end{aligned}
$$

Now, given $\varepsilon>0$, we can make the last term to be less than $\varepsilon / 3$ by taking $c$ large enough and using the equi-integrability of $\rho^{n}(x)$. Further, taking $K$ large enough we can ensure that the second term on the right-hand side of (7.5) is less than $\varepsilon / 3$, so that taking $|\Delta|<\varepsilon /(3 A)$ yields

$$
\int_{\Delta} f_{-}^{n}\left|v \cdot n_{x}\right| d \sigma_{x} d v<\varepsilon
$$

This proves that the sequence $f_{-}^{n}$ is weakly compact in $L^{1}\left(\partial \Omega^{-},\left|v \cdot n_{x}\right| d \sigma_{x} d v\right)$

Proof of Theorem 2.2. We take the weak form (7.1) in which we first consider $\psi$ with $\psi_{ \pm}=0$, so that the first two terms in (7.1) vanish. Passing to the limit in (7.1) for such $\psi$ is sufficient to obtain that $f$ is a mild solution of (2.1), namely that it satisfies (2.23) for almost all characteristics. Moreover, we can fix $R>0$ and for every $\varepsilon>0$ take $\psi=\psi_{\varepsilon}$ vanishing on a set of 
characteristics of measure $\varepsilon$ in $\partial \Omega_{R}^{-}$. In particular, by taking the sets $E_{\varepsilon, R}$ from Lemma 7.1, we can ensure that for every $\varepsilon>0, \chi_{\varepsilon} f^{n} v_{n}\left(f^{n}\right)$ and $\chi_{\varepsilon} Q_{n}^{+}\left(f^{n}, f^{n}\right)$ are weakly compact in $L^{1}\left(\Omega \times \mathbb{R}^{3}\right)$.

We next study the limits of each term in (7.1). By the weak convergence of $f^{n}$, the first term on the right-hand side converges to

$$
\iint_{\Omega \times \mathbb{R}^{3}} f v \cdot \nabla_{x} \psi_{\varepsilon} d x d v
$$

Further, since $v_{n}\left(f^{n}\right) \rightarrow v(f)$ strongly in $L_{\text {loc }}^{1}\left(\Omega \times \mathbb{R}^{3}\right)$, we can take a system of sets $E_{\eta}$ in $\Omega \times\{|v| \leq R\}$, with the complements of measure $\eta$ and the characteristic functions $\chi_{\eta}$, such that for all $\eta>0$, the sequence $\chi_{\eta} v_{n}\left(f^{n}\right)$ converges uniformly in $(x, v)$ to a bounded limit $\chi_{\eta} v(f)$. Therefore, keeping $\varepsilon$ and $\eta$ fixed, we obtain by the weak convergence of $f^{n}$,

$$
\lim _{n \rightarrow \infty} \iint_{\Omega \times \mathbb{R}^{3}} f^{n}\left(v_{n}\left(f^{n}\right)+\alpha_{n}\right) \psi_{\varepsilon} \chi_{\eta} d x d v=\iint_{\Omega \times \mathbb{R}^{3}} f v(f) \psi_{\varepsilon} \chi_{\eta} d x d v .
$$

By the weak compactness of $f^{n} v_{n}\left(f^{n}\right) \psi_{\varepsilon}$, it follows that $f v(f) \psi_{\varepsilon} \in L^{1}$, so that we can remove the $\eta$ truncation and pass to the limit in the 'loss' term with $\psi_{\varepsilon}$. It remains to study the limit of the 'gain' term in the integral form (7.1), and to prove that

$$
\lim _{n \rightarrow \infty} \iint_{\Omega \times \mathbb{R}^{3}} Q_{n}^{+}\left(f^{n}, f^{n}\right) \psi_{\varepsilon} d x d v=\iint_{\Omega \times \mathbb{R}^{3}} Q^{+}(f, f) \psi_{\varepsilon} d x d v .
$$

Without restricting generality, we can assume that the test functions $\psi_{\varepsilon}$ in (7.6) are nonnegative. Taking a positive constant $M$ we truncate the 'gain' term $Q_{n}^{+}\left(f^{n}, f^{n}\right)$, replacing its first argument by $\min \left(f^{n}, M\right)$. The nonnegative correction term is estimated with the help of (4.18):

$$
\begin{aligned}
& \iint_{\Omega \times \mathbb{R}^{3}}\left(Q_{n}^{+}\left(f^{n}, f^{n}\right)-Q_{n}^{+}\left(\min \left(f^{n}, M\right), f^{n}\right)\right) \psi_{\varepsilon} d x d v \\
\leq & \frac{C}{\log K}+K \iint_{\left\{f^{n}>M\right\}} f^{n} v_{n}\left(f^{n}\right) \psi_{\varepsilon} d x d v \leq \frac{C}{\log K}+K \varepsilon_{1}(M)
\end{aligned}
$$

(for $K>1$ large enough, so that the factor in front of the entropy dissipation in (4.18) is bounded above by $C / \log (K))$. Here $\varepsilon_{1}(M)$ tends to zero as $M \rightarrow \infty$ uniformly in $n$ because of the weak compactness of $f^{n} v_{n}\left(f^{n}\right) \psi_{\varepsilon}$. Next, we take a positive $\lambda$ and define the truncated 'gain' term $Q_{n \lambda}^{+}$by multiplying the integrand in $Q_{n}^{+}\left(\min \left(f^{n}, M\right), f^{n}\right)$ by $\chi_{\lambda}=\chi_{\left\{|v|^{2}+\left|v_{*}\right|^{2}<\lambda\right\}}$. For $M$ fixed, the following estimate for the nonnegative difference term is obtained by using (4.18):

$$
\begin{aligned}
& \iint_{\Omega \times \mathbb{R}^{3}}\left(Q_{n}^{+}\left(\min \left(f^{n}, M\right), f^{n}\right)-Q_{n \lambda}^{+}\left(\min \left(f^{n}, M\right), f^{n}\right)\right) \psi_{\varepsilon} d x d v \\
= & \iint_{\left.\Omega \times \mathbb{R}^{3}\right)^{2}} \min \left(f^{n \prime}, M\right) f_{*}^{n \prime} B_{n}\left(1-\chi_{\lambda}\right) \psi_{\varepsilon} d x d v d y d v_{*} \\
\leq & \frac{C}{\log K}+K M \varepsilon_{2}(\lambda),
\end{aligned}
$$


where $\varepsilon_{2}(\lambda) \rightarrow 0$ as $\lambda \rightarrow \infty$ uniformly in $n$. Using the change of variables $\left(v^{\prime}, v_{*}^{\prime}\right) \mapsto\left(v, v_{*}\right)$, the integral of the truncated 'gain' term can be written as follows:

$$
\begin{aligned}
& \iint_{\Omega \times \mathbb{R}^{3}} Q_{n \lambda}^{+}\left(\min \left(f^{n}, M\right), f^{n}\right) \psi_{\varepsilon} d x d v \\
= & \iint_{\Omega \times \mathbb{R}^{3}} d x d v \min \left(f^{n}, M\right) \iint_{\Omega \times \mathbb{R}^{3}} d y d v_{*} f_{*}^{n} B_{n} \chi_{\lambda} \psi_{\varepsilon}^{\prime} .
\end{aligned}
$$

Now by the weak convergence of $f^{n}$,

$$
\iint_{\Omega \times \mathbb{R}^{3}} d y d v_{*} f_{*}^{n} B_{n} \chi_{\lambda} \psi_{\varepsilon}^{\prime} \underset{n \rightarrow \infty}{\longrightarrow} \iint_{\Omega \times \mathbb{R}^{3}} d y d v_{*} f_{*} B \chi_{\lambda} \psi_{\varepsilon}^{\prime},
$$

strongly in $L^{1}$ and hence, for all $\eta>0$, uniformly on a set with complement of measure $\eta$ in the space of $(x, v)$. Denoting by $\chi_{\eta}$ the characteristic function of this set, we obtain

$$
\begin{aligned}
& \mid \iint_{\left(\Omega \times \mathbb{R}^{3}\right)^{2}} \min \left(f^{n^{\prime}}, M\right) f_{*}^{n^{\prime}} B_{n} \chi_{\lambda} \chi_{\eta} \psi_{\varepsilon} d x d v d y d v_{*} \\
& -\iint_{\left(\Omega \times \mathbb{R}^{3}\right)^{2}} f^{\prime} f_{*}^{\prime} B \chi_{\lambda} \chi_{\eta} \psi_{\varepsilon} d x d v d y d v_{*} \mid \leq \frac{C}{\log K}+K \varepsilon_{1}(M)+\varepsilon_{3}(\lambda, \eta, n),
\end{aligned}
$$

where $\varepsilon_{3}(\lambda, \eta, n) \rightarrow 0$ as $n \rightarrow \infty$ for fixed $\lambda$ and $\eta$, and $\varepsilon_{1}(M)$ is as above. The integral of $Q_{n \lambda}^{+}$over the exceptional set of measure $\eta$ can be estimated, analogously to (7.7), by

$$
\frac{C}{\log K}+K M \varepsilon_{4}(\lambda, \eta)
$$

where $\varepsilon_{4}(\lambda, \eta)$ tends to zero as $\eta \rightarrow 0$ for fixed $\lambda$, uniformly in $n$. Thus, we obtain

$$
\begin{aligned}
& \left|\iint_{\Omega \times \mathbb{R}^{3}} Q_{n}^{+}\left(f^{n}, f^{n}\right) \psi_{\varepsilon} d x d v-\iint_{\Omega \times \mathbb{R}^{3}} Q^{+}(f, f) \psi_{\varepsilon} d x d v\right| \\
\leq & \frac{C}{\log K}+K \varepsilon_{1}(M)+K M\left(\varepsilon_{2}(\lambda)+\varepsilon_{4}(\lambda, \eta)\right)+\varepsilon_{4}(\lambda, \eta, n) .
\end{aligned}
$$

From this (7.6) follows by letting $n \rightarrow \infty, \eta \rightarrow 0, \lambda \rightarrow \infty, M \rightarrow \infty$ and $K \rightarrow \infty$, in this order.

Thus, we have proved that $f$ satisfies the limit form of (7.1) with $\psi=\psi_{\varepsilon}$ vanishing on $\partial \Omega^{ \pm}$, and that $\chi_{\varepsilon} Q(f, f)$ is in $L^{1}\left(\Omega \times \mathbb{R}^{3}\right)$. It follows that $f$ is a mild solution of (2.1) and that $f^{\sharp}(s)$ is absolutely continuous for almost all characteristics. We can now get rid of the condition that the test functions $\psi$ vanish on the boundary by arguing as follows. Let $\psi$ be a function from 
$L^{\infty}\left(\Omega \times \mathbb{R}^{3}\right)$ such that $v \cdot \nabla_{x} \psi \in L^{\infty}\left(\Omega \times \mathbb{R}^{3}\right)$. The mild form (2.23) then implies, for almost all $(x, v) \in \partial \Omega^{-}$,

$$
f_{+} \psi_{+}-f_{-} \psi_{-}+\int_{-s^{+}(x, v)}^{0}\left(f v \cdot \nabla_{x} \psi+Q(f, f) \psi\right)^{\sharp} d s=0 .
$$

First of all, by taking $\psi$ vanishing on one of the ends of each characteristic, and integrating (7.8), it is easy to see that $\chi_{\varepsilon \pm} f_{ \pm} \in L^{1}\left(\partial \Omega^{ \pm},\left|v \cdot n_{x}\right| d \sigma_{x} d v\right)$. For such $\psi$, we can also pass to the limit in (7.1) with the test function $\chi_{\varepsilon} \psi$ and thereby obtain

$$
\lim _{n \rightarrow \infty} \iint_{\partial \Omega^{ \pm}} \chi_{\varepsilon} \psi_{ \pm} f_{ \pm}^{n}\left|v \cdot n_{x}\right| d \sigma_{x} d v=\iint_{\partial \Omega^{ \pm}} \chi_{\varepsilon} \psi_{ \pm} f_{ \pm}\left|v \cdot n_{x}\right| d \sigma_{x} d v
$$

Taking $\psi=1$ on $\partial \Omega^{ \pm}$and letting $\varepsilon \rightarrow 0$ and using that the integrals on the left-hand side of (7.9) are bounded uniformly in $n$, we obtain by the monotone convergence that $f_{ \pm} \in L^{1}\left(\left|v \cdot n_{x}\right| d \sigma_{x} d v\right)$. Using (7.8) we then obtain that $\int_{-s^{+}(x, v)}^{0} Q(f, f)^{\sharp} \psi d s$ is integrable over $\partial \Omega^{-}$and therefore, $f$ satisfies (2.30).

Using the weak compactness of $f_{ \pm}^{n}$ obtained as a consequence of Lemmas 3.2 and 7.2 we can then pass to the limit in the boundary condition satisfied by $f_{ \pm}^{n}$ :

$$
f_{+}^{n}=\mathcal{R} f_{-}^{n}+\alpha_{n} S \iint_{\Omega \times \mathbb{R}^{3}} f^{n} d x d v .
$$

Indeed, the convergence (7.9) then implies that

$$
f_{ \pm}^{n} \rightarrow f_{ \pm}, \text {weakly in } L^{1}\left(\partial \Omega^{ \pm},\left|v \cdot n_{x}\right| d \sigma_{x} d v\right) .
$$

Writing the boundary operator $\mathcal{R}$ in the weak form with $\psi \in L^{\infty}\left(\partial \Omega^{+}, \mid v\right.$. $\left.n_{x} \mid d \sigma_{x} d v\right)$ we have,

$$
\begin{aligned}
& \iint_{\partial \Omega^{+}} \mathcal{R} f^{n} \psi\left|v \cdot n_{x}\right| d \sigma_{x} d v \\
= & \iint_{\partial \Omega^{-}} d \sigma_{x} d w\left|w \cdot n_{x}\right| f_{-}^{n} \int_{v \cdot n_{x}>0} k(x, v, w) \psi d v .
\end{aligned}
$$

Using condition (2.16) and the upper bound (2.19), we can pass to the limit as $n \rightarrow \infty$ in (7.11) obtaining

$$
\mathcal{R} f_{-}^{n} \rightarrow \mathcal{R} f_{-} \text {, weakly in } L^{1}\left(\partial \Omega^{+},\left|v \cdot n_{x}\right| d \sigma_{x} d v\right) .
$$

The last term in (7.10) converges to zero uniformly in $(x . v) \in \partial \Omega^{+}$and decays rapidly for $|v|$ large. Passing to the weak limit in (7.10) we obtain the boundary condition almost everywhere on $\partial \Omega^{+}$.

Finally, we notice that taking the test functions $\psi$ growing as $1+|v|^{2}$ for $|v|$ large, by using the uniform bounds for the energy and mass of the approximate solutions and the monotone convergence theorem, we can establish that the limit solutions $f$ and their boundary traces are also integrable with the weight $\left(1+|v|^{2}\right)$. This will complete the proof of the theorem. 
We finally give a proof of Theorem 2.3, which will be constructed by analogy with the already treated reflection case. A number of arguments can be simplified in view of the assumed bounds for the entropy inflow (2.21). Without restricting generality we will assume that the boundary profile $f_{b}$ satisfies the condition $\iint_{\partial \Omega^{+}} f_{b}\left|v \cdot n_{x}\right| d \sigma_{x} d v=1$.

Proof of TheOREM 2.3. Take positive parameters $r, m, \alpha, j$ and $l$ and consider the linear-type problem (the nonlinearity is introduced by the cutoffs) for $F=F^{n}$, where $n=(r, m, \alpha, j, l)$ :

$$
\begin{aligned}
v \cdot \nabla_{x} F+\alpha F & =Q_{n}^{+}(F, f)-v_{n}(f) F, & & (x, v) \in \Omega \times \mathbb{R}^{3}, \\
F_{+} & =f_{b}, & & (x, v) \in \partial \Omega^{+}
\end{aligned}
$$

with truncations and regularizations as in Section 4 (except for the boundary regularization which is not needed). The unique solution of this problem is obtained as the limit of the sequence of iterations analogous to (4.7). This allows us to define the operator

$$
T: f \mapsto k F / \mu(F)
$$

which maps the convex bounded set $L=\left\{f \in L^{1} \mid \mu(f)=\kappa\right\}$ into itself. Following the arguments in Section 4 we verify the continuity and compactness of $T$ and find a fixed point by Schauder's theorem. We then pass to the limit as $l \rightarrow \infty$ and as $j \rightarrow \infty$ using the strong and weak compactness of solutions, guaranteed by the positiveness of $\alpha$. The limit function $F$ will then satisfy equation (3.12) with the $r$ and $m$ truncations and the boundary condition $F_{+}=f_{b}$. Because of the uniform control over the mass and energy fluxes, the entropy production term

$$
\frac{1}{\mu(F)} \iint_{\left(\Omega \times \mathbb{R}^{3}\right)^{2}}\left(F F_{*}-F^{\prime} F_{*}^{\prime}\right) \log \frac{F F_{*}}{F^{\prime} F_{*}^{\prime}} B_{r m} d x d v d y d v_{*}
$$

is bounded uniformly in $r, m$ and $\alpha$. In view of the lower bound assumed in the statement of the theorem, the proof of Lemma 6.1 holds. By Lemma 6.3 and the uniform energy bound, $\mu(F)$ is uniformly bounded from above. Also by (5.10) and Lemma 5.3, it is uniformly bounded from below. Multiplication by $\kappa / \mu(F)$ gives the equations for the normalized function $f$ satisfying $\mu(f)=\kappa$ :

$$
\begin{aligned}
v \cdot \nabla_{x} f+\alpha f & =Q(f, f), & & (x, v) \in \Omega \times \mathbb{R}^{3}, \\
f_{+} & =\gamma f_{b}, & & (x, v) \in \partial \Omega^{+},
\end{aligned}
$$

where $\gamma=\kappa / \mu(F)$. As in Section 4, $Q=Q_{r m}$ is the collision operator with the truncated kernel $B_{r m}$ and the constant $\lambda$ is uniformly bounded from above and from below. The uniform energy estimate implies that $\int_{|v|>R} f(x, v) d x d v$ 
are uniformly small if $R$ is large enough. We can then use inequality (2.25) to estimate the entropy of solutions $f$ :

$$
\begin{aligned}
& \int_{\left\{|v|>\delta ; \int v^{\sharp} d s \leq a\right\}} f \log f d x d v \leq \int_{\left\{|v|>\delta ; \int \nu^{\sharp} d s \leq a ; f>1\right\}} f \log f d x d v \\
\leq & \frac{D}{\delta} e^{a / \delta} \iint_{\partial \Omega^{-}} f_{-}\left(a / \delta+\left|\log f_{-}\right|\right)\left|v \cdot n_{x}\right| d \sigma_{x} d v .
\end{aligned}
$$

Since $\mu(f)=\kappa$, the bound (2.27) implies that for every approximating solution $f$, the set $\left\{(x, v)|| v \mid \leq R, \int_{-s^{+}}^{s^{-}} v(f)^{\sharp} d s>a\right\}$ has uniformly small $v$-sections. Thus, by Lemma 6.4 , choosing $\delta$ small and $a$ large enough, the integral

$$
\int_{\left\{|v| \leq \delta \text { or } \int \nu^{\sharp} d s>a\right\}} f(x, v) d x d v
$$

can be made as small as desired for all $f$ from the family of solutions. The estimate of the $f \log f$ term on the remainder set then implies the weak compactness of $f$.

For 'hard' interactions, we can then conclude as in the proof of Theorem 2.1. For 'soft' interactions we continue along the lines of the proof of Theorem 2.2. Notice the the uniform integrability of the boundary trace $f_{-}^{n}$ implied by the entropy estimate can be used to drastically shorten the proof of Lemma 7.1, reducing it to a single estimate of the type (7.3). Passing to the limit in the boundary condition

$$
f_{+}^{n}=\lambda_{n} f_{b}
$$

using (7.9), we obtain that the limit trace satisfies the equality $f_{+}=\lambda f_{b}$ with $\gamma$ in the compact interval defined by the upper and lower bounds of $\kappa / \mu(F)$.

\section{Conclusions}

We established the existence of $L^{1}$ solutions to the stationary Povzner equation (2.1). A number of related questions seem to be worth mentioning in this context. First of all, it is likely that several assumptions made in the paper are of purely technical character and could be removed in a subsequent development. For instance, an extension of the present results to domains with less regular boundaries and collision kernels $B$ in (2.7) seem feasible (the precise factorization form in (2.7) is also taken for convenience rather than out of necessity). The natural questions of uniqueness and further regularity of the obtained solutions are certainly more deep and seem to require substantial new ideas. 
Another natural question is the possibility of the localization of the kernel $b(x, y)$ in (2.7) and the corresponding limit transition to the Enskog and Boltzmann equations, as described formally by (2.11). The former equation seems to be especially closely connected to the present analysis, and in particular, estimates based on the properties of the collision frequency should remain uniform with respect to the localization parameter when passing from (2.7) to (2.11). Indeed, the Enskog collision frequency

$$
v(f)(x, v)=\sigma^{2} \int_{\mathbb{R}^{3}} \int_{S^{2}} f\left(x+\sigma \omega, v_{*}\right) \chi_{\left\{\left(v-v_{*}\right) \cdot \omega<0\right\}}\left|\left(v-v_{*}\right) \cdot \omega\right| d \omega d v_{*}
$$

can be shown to satisfy the estimate

$$
\sup _{x \in \partial \Omega} \int_{S_{+}^{2}} \sup _{|v| \leq R}\left\{|v| \int_{-s^{+}(x, v)}^{s^{-}(x, v)} v(f)^{\sharp} d s\right\} d \eta \leq C_{R} \iint_{\Omega \times \mathbb{R}^{3}} f_{*}\left(1+\left|v_{*}\right|\right) d y d v_{*},
$$

where $v=\eta|v|$ and $S_{+}^{2}=\left\{\eta \in S^{2} \mid\left(\eta \cdot n_{x}\right)>0\right\}$, and a similar bound for $x \in \Omega$. Thus, Assumption 5.1 can be verified in the case of the Enskog equation, and the lower bound analysis of Section 5 carries over. The bottleneck seems to be the argument in Lemma 6.2, which relies on the extra integration in the Povzner collision operator. Nevertheless, it is possible that a more detailed analysis of the spatial regularity of solutions in the Enskog case could allow one to prove the weak $L^{1}$ compactness of solutions using the general framework of Section 6 .

\section{REFERENCES}

[1] L. ARKeryd, On the stationary Boltzmann equation in $\mathbb{R}^{n}$, Internat. Math. Res. Notices (2000), 625-641.

[2] L. Arkeryd - C. Cercignani, On the convergence of solutions of the Enskog equation to solutions of the Boltzmann equation, Comm. Partial Differential Equations 14 (1989), 1071-1089.

[3] L. Arkeryd - C. Cercignani, Global existence in $L^{1}$ for the Enskog equation and convergence of the solutions to solutions of the Boltzmann equation, J. Statist. Phys. 59 (1990), 845-867.

[4] L. Arkeryd - C. Cercignani - R. Illner, Measure solutions of the steady Boltzmann equation in a slab, Comm. Math. Phys. 142 (1991), 285-296.

[5] L. ARKERYD - A. NOURI, A compactness result related to the stationary Boltzmann equation in a slab, with applications to the existence theory, Indiana Univ. Math. J. 44 (1995), 815839.

[6] L. ARKERYD - A. Nouri, The stationary Boltzmann equation in the slab with given weighted mass for hard and soft forces, Ann. Scuola Norm. Sup. Pisa Cl. Sci. (4) 27 (1998), 533-556.

[7] L. ARKeryd - A. Nouri, On the stationary Povzner equation in $\mathbb{R}^{n}$, J. Math. Kyoto Univ. 39 (1999), 115-153. 
[8] L. Arkeryd - A. Nouri, $L^{1}$ solutions to the stationary Boltzmann equation in a slab, Ann. Fac. Sci. Toulouse Math. (6) 9 (2000), 375-413.

[9] L. ARKERYD - A. Nouri, The stationary Boltzmann equation in $\mathbb{R}^{n}$ with given indata, Ann. Sc. Norm. Super. Pisa Cl. Sci. (5) 1 (2002), 359-385.

[10] P. Broman, On a class of boundary value problems for the Povzner equation, preprint no. 1993:27, Chalmers Univ. Tech., Göteborg (1993).

[11] S. Caprino - M. Pulvirenti - W. Wagner, Stationary particle systems approximating stationary solutions to the Boltzmann equation, SIAM J. Math. Anal. 29 (1998), 913-934.

[12] C. Cercignani, The Grad limit for a system of soft spheres, Comm. Pure Appl. Math. 36 (1983), 479-494.

[13] C. Cercignani, Measure solutions for the steady nonlinear Boltzmann equation in a slab, Comm. Math. Phys. 197 (1998), 199-210.

[14] C. Cercignani - R. Illner - M. Pulvirenti, "The mathematical theory of dilute gases", Springer-Verlag, New York, 1994.

[15] C. Cercignani - R. Illner - M. Pulvirenti - M. Shinbrot, On nonlinear stationary half-space problems in discrete kinetic theory, J. Statist. Phys. 52 (1988), 885-896.

[16] C. Cercignani - R. Illner - M. Shinbrot, A boundary value problem for the twodimensional Broadwell model, Comm. Math. Phys. 114 (1988), 687-698.

[17] J. Darrozes - J.-P. Guiraud, Généralisation formelle du théorème $H$ en présence de parois. Applications, C. R. Acad. Sc. Paris 262 (1966), 1368-1371.

[18] R. DiPerna - P. L. Lions, On the Cauchy problem for the Boltzmann equation: Global existence and weak stability, Ann. of Math. 130 (1989), 321-366.

[19] M. J. Esteban - B. Perthame, On the modified Enskog equation for elastic and inelastic collisions. Models with spin, Ann. Inst. H. Poincaré Anal. Non Linéaire 8 (1991), 289-308.

[20] F. Golse - L. SAint-RAYmond, Velocity averaging in $L^{1}$ for the transport equation, C. R. Math. Acad. Sci. Paris 334 (2002), 557-562.

[21] J.-P. Guiraud, Problème aux limites intérieur pour l'équation de Boltzmann en régime stationnaire, faiblement non linéaire, J. Mécanique 11 (1972), 183-231.

[22] N. M. GüNTER, "Potential theory and its applications to basic problems of mathematical physics", Frederick Ungar Publ. Co., New York, 1967.

[23] A. HeInTz, Initial-boundary value problems in irregular domains for nonlinear kinetic equations of Boltzmann type, Transport Theory Statist. Phys. 28 (1999), 105-134.

[24] R. Illner - J. STRuckmeier, Boundary value problems for the steady Boltzmann equation, J. Statist. Phys. 85 (1996), 427-454.

[25] A. I. KhisAmutdinov, A simulation method for statistical modeling of rarefied gases, Dokl. Akad. Nauk SSSR 291 (1986), 1300-1304.

[26] M. Lachowicz - M. Pulvirenti, A stochastic system of particles modelling the Euler equations, Arch. Ration. Mech. Anal. 109 (1990), 81-93.

[27] N. B. Maslova, Stationary problems for the Boltzmann equation in the case of large Knudsen numbers, Dokl. Akad. Nauk SSSR 229 (1976), 593-596.

[28] N. B. Maslova, "Nonlinear evolution equations. Kinetic approach", vol. 10 of Series on Advances in Mathematics for Applied Sciences, World Scientific Publishing Co. Inc., River Edge, NJ, 1993.

[29] S. MisCHLER, On weak-weak convergences and applications to the initial-boundary value problems for kinetic equations, preprint no. 35 of the University of Versailles, (1999). 
[30] A. Nouri, Private communication, Dec., 1999.

[31] V. A. PAnferov, Two problems on existence and approximation related to the Boltzmann equation, Ph. D. thesis, Chalmers University of Technology, Göteborg, Sweden, 1999.

[32] Y.-P. PAO, Boundary-value problems for the linearized and weakly nonlinear Boltzmann equation, J. Mathematical Phys. 8 (1967), 1893-1898.

[33] A. Y. Povzner, On the Boltzmann equation in the kinetic theory of gases, Mat. Sbornik (N. S.) 58/100 (1962), 65-86. Translated in Amer. Math. Soc. Transl., Ser. 2, 47, pp. 193-216, AMS, Providence RI, (1965).

[34] P. RÉsiboIs, $H$ theorem for the (modified) nonlinear Enskog equation, J. Statist. Phys. 19 (1978), 593-609.

[35] P. Résibois - M. De LeEner, "Classical Kinetic Theory of Fluids", John Wiley \& Sons, New York, 1977.

[36] S. UKaI, Solutions of the Boltzmann equation, in Patterns and waves, North-Holland, Amsterdam (1986), 37-96.

[37] S. Ukai - K. Asano, Steady solutions of the Boltzmann equation for a gas flow past an obstacle. I. Existence, Arch. Ration. Mech. Anal. 84 (1983), 249-291.

[38] W. WAGNER, A convergence proof for Bird's direct simulation Monte Carlo method for the Boltzmann equation, J. Statist. Phys. 66 (1992), 1011-1044.

Department of Mathematics and Statistics McMaster University

Hamilton, Ontario

L8S 4K1 Canada

panferov@math.mcmaster.ca 